\title{
Tensile Structures of Cables Net, Guidelines to Design and Applications
}

\author{
Fabio Rizzo \\ Department of Engineering and Geology, G. D’Annunzio University of Pescara, Pescara, Italy \\ Email: fabiorizzo.unich@gmail.com
}

Received 9 December 2015; accepted 27 March 2016; published 30 March 2016

Copyright (C) 2016 by author and Scientific Research Publishing Inc. This work is licensed under the Creative Commons Attribution International License (CC BY). http://creativecommons.org/licenses/by/4.0/ (c) (i) Open Access

\section{Abstract}

The structural engineering design of not conventional typologies imposes a complex path that begins evaluating procedures of a preliminary design and ends with complex procedures to validate the analysis response. Any guide lines to follow are often available. About complex shapes, in particular, any details are presented in the codes to evaluate wind action and so wind tunnel experiments are necessary to valuate this. The evaluation of wind tunnel data is a complex process that often needs new and specific subroutines programmed by researchers. The difficult increases when the objective is to study a not specific building but general aspects as for examples the dependence of a generic phenomenon by a geometric sample; in this case it is necessary to design and to program numerical subroutines before and then the wind tunnel experiments. Often, these subroutines are left detached and are non-generalizable process. Purpose of this paper is to describe a complete procedure to pre- and post-process wind tunnel data with the objective to design a not convectional structure as a tensile structure. In this particular case the research aim is a parametrization of the aerodynamic behavior of Hyperbolic Paraboloid roofs, shape used for cables net. The reason of the experiments is the absence in the international codes of the pressure coefficients for these geometries. The paper describes the numerical procedure evaluated to choose a sufficient representative geometric sample, the numerical procedure evaluated to design and to construct the wind tunnel models and FE models, the numerical procedure to evaluate and to use for FEM analyses of the wind tunnel data, the numerical procedure to calculate nonlinear structural analysis, and, finally some applications. All these numerical procedures use basic theory derived for example by the cable theory, the fluid mechanic, the nonlinear geometric analysis and other. However specific codes were necessary and were programmed to apply the theories on the specific case of study; the complete methodology followed is presented. The goal is to create a free open domain where the numerical procedures evaluated are merged, added, modified by researchers with the aim to obtain a common space of use for wind engineering of not conventional structure. 


\section{Keywords} Tensile Structure, Numerical Procedure, Tensile Structures, Cables Net Preliminary Design,
Tensile Mesh Generator, Wind Tunnel Testing

\section{Introduction}

The structural engineering is an open space where a great number of different needs converge in order to obtain a one solution. The high performance is often obtained using traditional structural typologies but increasing the element sections dimensions. Often the solution is to use different systems and typologies. Cables structures for example are particularly adapted to design large span but they need particular structures and shapes. This structural typology isn't presented in the technical codes and also a preliminary design is impossible without a specific study. The particular shape isn't presented in the wind action section of the codes and so is impossible to evaluate the wind action also for sample structures that use this system. Wind tunnel tests should be carried out. The wind tunnel experiments are often performed for a specific building and are used to study the wind-structure interaction in order to the design the building structures. However, in many cases the wind tunnel is a very important instrument of research. It's important sometimes to study preliminarily a phenomenon in order to have a parametrization of the aerodynamic behavior. In both cases many numerical subroutines are programmed by the researchers to prepare the wind tunnel setup and to evaluate the wind tunnel experiments. Often, these numerical procedures are left detached and are created for the specific case studied. If the purpose of the research is a parametrization of a phenomenon it's interesting to program codes generalizable in order to extend the research with a great number of different cases. Purpose of this paper is to describe a complete process of pre- and postprocession of wind tunnel data acquired with experiments to look for a parametrization of the aerodynamic behavior of a specific geometry: in this case the hyperbolic paraboloid geometry was studied. The objective is double: at first to give an example to follow in order to start a parametric experimental campaign, at second to give the possibility to extend the research. To obtain these results, the four different numerical procedures programmed will be described and the basic theory following will be summarized. In Section 2, the first numerical procedure to look for a sufficient representative geometric sample is presented. In Section 3, a numerical procedure programmed to obtain FEM three-dimensional models is proposed. In Section 4, the procedure to evaluate the wind tunnel acquisitions is summarized and in particular in Section 4.2.3, the application of the wind tunnel data to perform FEM analysis is described. Finally in Section 5, a research nonlinear structural analysis program is described to close the pre- and post-processing methodology. The results are a general main procedure to use for each similar research and also that can be used to extend this particular research with other geometries. The educational purposes of the paper want to sustain the research based on a personal idea.

\section{Numerical Procedure for Preliminary Design Cables Net}

The example of pre and post procedure to perform wind tunnel data consists in a research focused on the hyperbolic paraboloid geometry. The purpose of this research conducted by the Department of engineering and geology of Pescara (Italy) University and by the CRIACIV (Interuniversity Centre for Building Aerodynamics and Wind Engineering) wind tunnel laboratory, is to obtain a parametrization of the aerodynamic behavior of the hyperbolic paraboloid roofs (Rizzo F., et al., 2011; Rizzo F., et al., 2012; Rizzo F., 2012; Rizzo F., Sepe V., 2015). This particular shape is used to build tensile structures to cover for example sports arena, meeting or conference rooms. In the international codes there aren't information about wind loads and in particular aren't pressure coefficients to use as reference (ASCE 2005; AS/NZS 2002; CNR-DT 207/2008; CEN 2005). The difficulty to start a similar research is the little information this kind of structures; the wind tunnel tests are often performed for specific cases and are not generalizable; the first phase of the work is focused to look for a geometric sample to test in wind tunnel. The ratio between geometry and structural performances is very important for this kind of structures. The Hyperbolic paraboloid surface is characterized by four geometrical parameters: the sags and the spans of the two orders of parabola generating. In order to study the ratio between these four geometrical parameters and the structural response a numerical procedure is necessary [1]-[7]. 


\subsection{Main Program}

Hyperbolic paraboloid cables net have a double curvature with different cable lengths and curvatures, and generally, different cable areas and pre-stresses. In addition, the shape plays a decisive role in the cables net deformation behavior under the action of external loads. In the cables net with opposite curvature, the two orders of cables become load-bearing or stabilizers depending on the direction of the acting load; the load-bearing cable is concave in the direction of the acting load. Therefore, in conditions of snow or wind suction, the two orders of cables reverse their curvature. This inversion is dangerous because it may lead to the instability of either the cables net or the border structures. The function adopted to describe the hyperbolic paraboloid is expressed by Equation (1), where $x, y$ and $z$ are respectively the spatial variables; $x_{0}, y_{0}$ and $z_{0}$ are the coordinates of the origin of the axes $a, b$ and $c$ are the geometric coefficients of the function. The $c$ parameter was set equal to 1 , making all the parabolas that lying on the surface, parallel and of identical curvature.

$$
\frac{\left(X-x_{0}\right)^{2}}{a^{2}}-\frac{\left(Y-y_{0}\right)^{2}}{b^{2}}=\frac{\left(Z-Z_{0}\right)}{c} \text { where } 1 / c=1
$$

It's important to precise that the initial geometry is only an initial condition and that the real geometry of the net is defined on bases of the cables pre-stress. The loads effect modifies again the geometry and the objective of a correct design is to minimize the geometry initial variation with and without the pre-stress and the loads. The numerical procedure programmed starts from a fixed geometrical configuration and evaluates the final geometrical configurations with the pre-stress or with the other loads considered. The pre-sizing procedure implemented has the aim of obtaining pre-stress values and initial cables areas which permit an optimal structural response with a final geometry similar to the fixed geometry. A small difference between the fixed geometry and the deformed shape is important because the pressure coefficients evaluated in wind tunnel are valid for each load cases.

Geometries that allow an optimal configuration and a good relationship between shape and structural performance are identified investigating a set of about thousand different geometric configurations and extrapolating those which, for the same geometry, provide the best structural performance in terms of cable area and cables stresses, and therefore, in terms of structural weight and lower displacements in operating conditions.

In order to create a numerical procedure, the real three-dimensional case was simplified with a two-dimensional model called "Rope beam", like illustrated in Figure 1. The two-dimensional structural model at the base of the

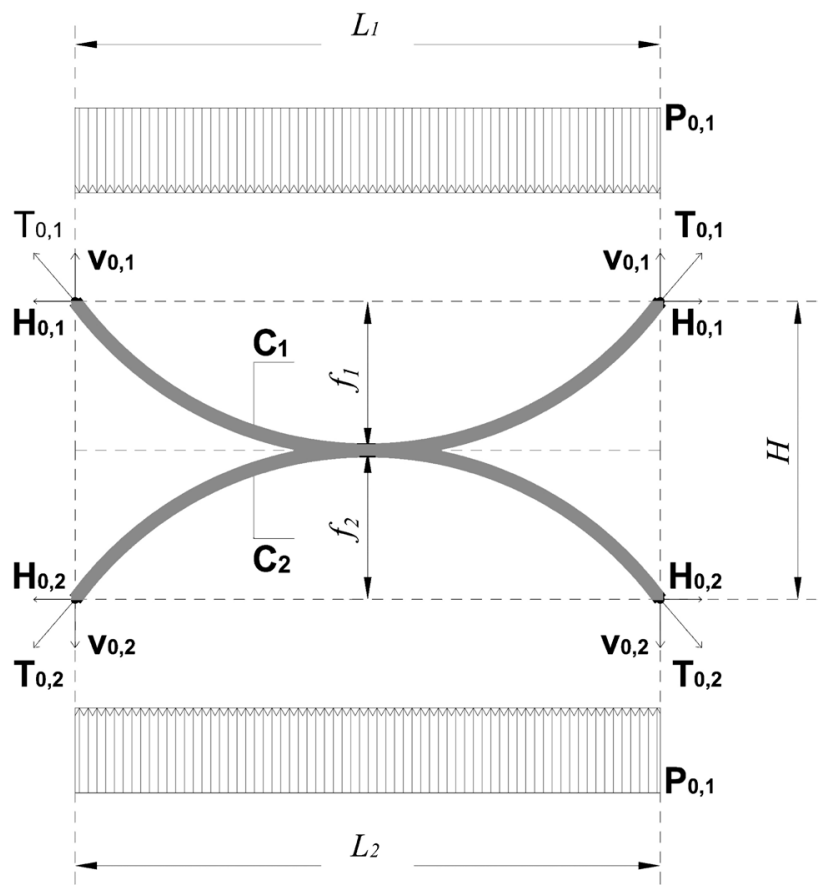

Figure 1. Rope beam, 2D structural system. 
proposed procedure consists of two cables with opposite curvature which have a node at midspan in common. The equilibrium of the system forces in the node in common ensures the forces transmission between the two cables.

The prefixed assumptions are: vertical links, in tension or in compression, are treated as a continuous membrane between the two main cables; the horizontal displacements are neglected compared to the vertical ones; pretension is considered as an equivalent distributed load; the system congruence is required only in the central span node; the mutual actions between cables are uniformly distributed as the external load, and consequently, load-bearing and stabilizing cable have a parabolic configuration also in elastic regime.

The stiffness coefficients are considered, respectively for the load-bearing cable $\left(C_{1}\right)$ and stabilizing one $\left(C_{2}\right)$ defined as expressed in Equation (2) and Equation (3), where $k_{1}$ and $k_{2}$ represent, respectively, the stiffness of the load-bearing cable $C_{1}$ and the stiffness of the stabilizing cable $C_{2}$, defined in Equation (4); $A_{1}$ and $A_{2}, f_{1}$ and $f_{2}, L_{1}$ and $L_{2}$ are, respectively, area, sag and span length of the cable $C_{1}$ and $C_{2}$.

According to the iterative numerical procedure, given a fixed geometry, the external loads, the maximum stress limits and, finally, the maximum value of the forces to be transmitted to the support structures in conditions of maximum load applied, it is possible to compute the optimal cables area.

Cables areas are considered optimal if the cables stresses, under the maximum load design, respectively of compression and aspiration, are close to the upper and lower limit fixed in the hypothesis $\left(\sigma_{\max }=1700.0 \mathrm{MPa}\right.$ and $\sigma_{\min }=20.0 \mathrm{MPa}$ ). The global flow chart of the numerical procedure for cables net preliminary design is shown in Figure 2. It consist of two sub-procedures, respectively the procedure 1, illustrated in Figure 3, which allows to determine the optimum area of cable $C_{1}$, and the procedure 2, illustrated in Figure 4, which allows to compute the area of cable $\mathrm{C}_{2}$ and therefore, the balance of the applied loads, depending on the area of cable $C_{1}$.

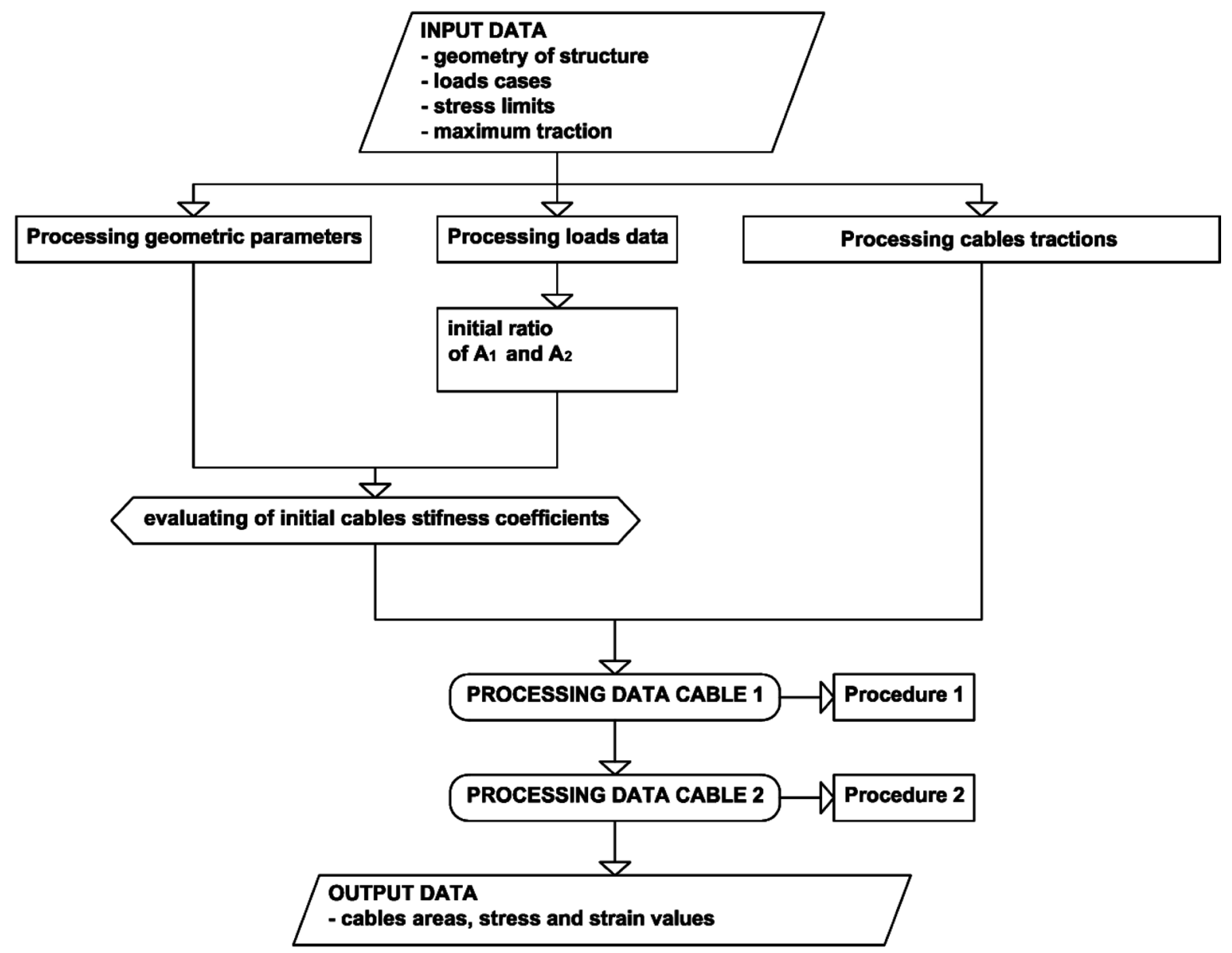

Figure 2. Numerical procedure for cables net preliminary design, global flow chart. 


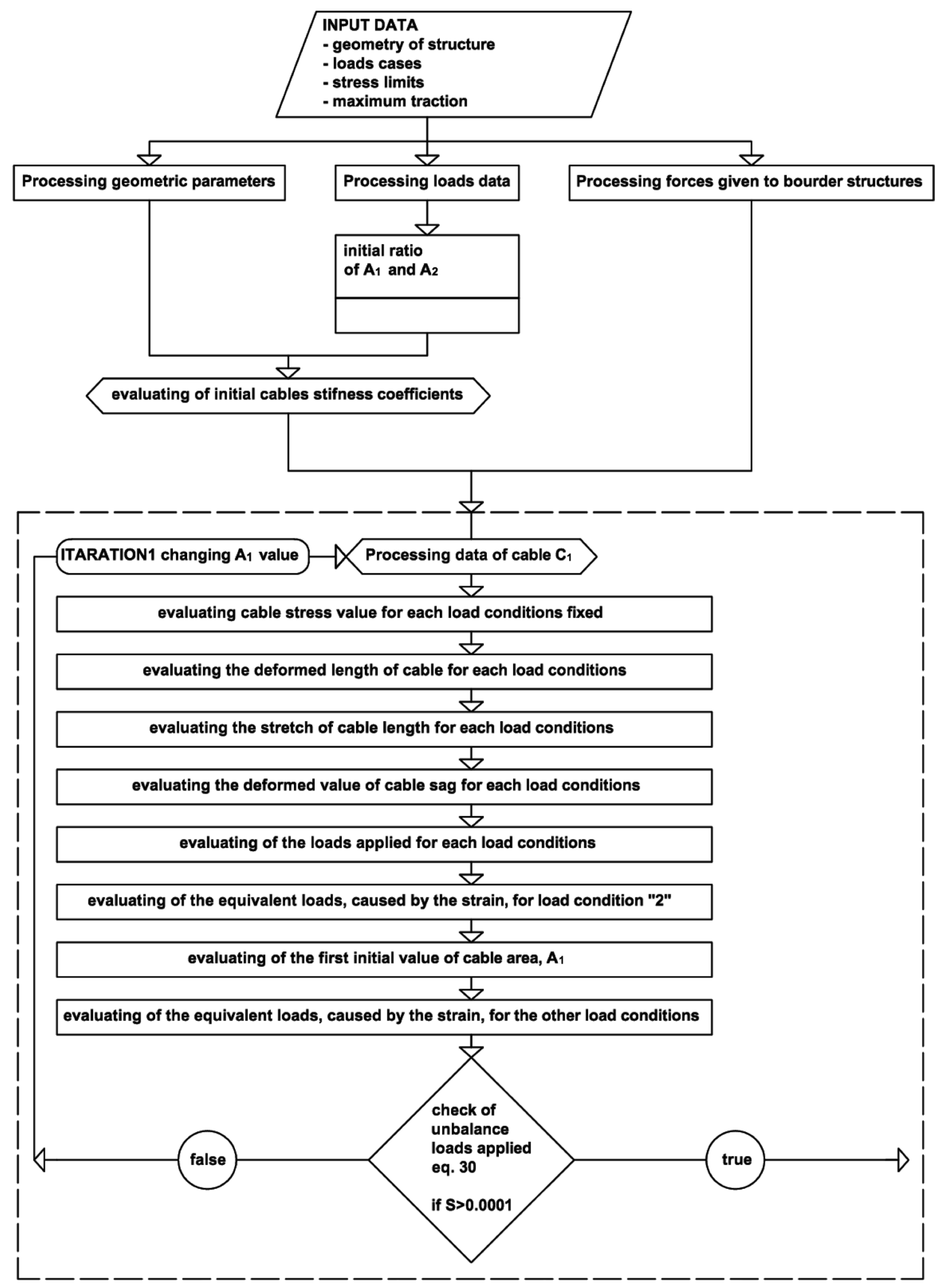

Figure 3. Numerical procedure to preliminary design load bearing cables of a cables net. 


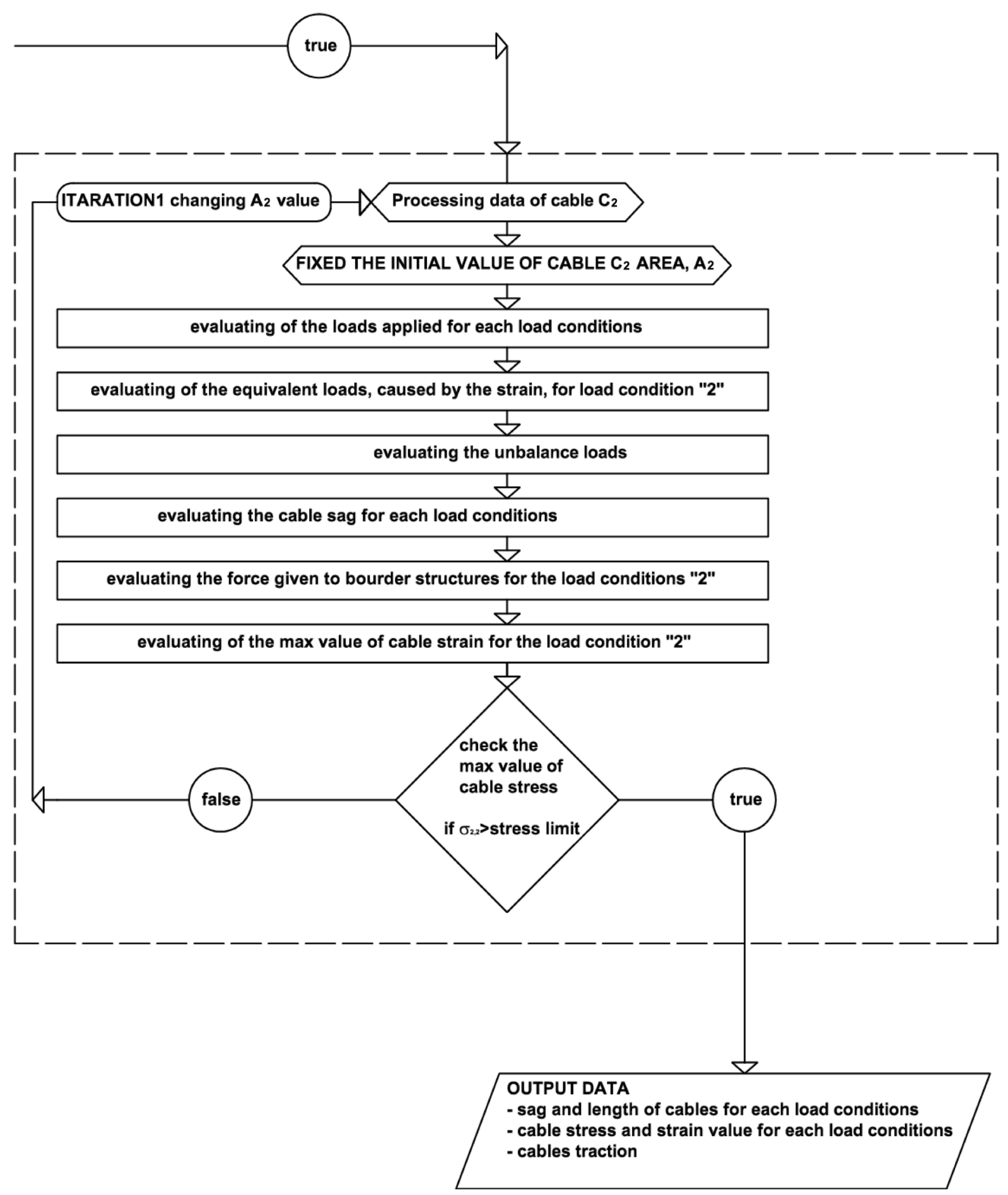

Figure 4. Numerical procedure to preliminary design stabilizing cables of a cables net.

With reference to Figure 1, the prefixed geometric quantities are the cable sags $\left(f_{1}\right.$ and $\left.f_{2}\right)$ and the span length ( $L_{1}$ and $L_{2}$ ). Subscripts 1 and 2 indicate, respectively, load-bearing cables and stabilizing one. The considered load configurations are: initial equilibrium ("0"), in which acts only the cables net self-weight and in which the internal cables tensions are induced from initial pre-stress; application of permanent loads (“1”), in which, in addition to the cables net self-weight, acts membranes and/or roof panels’ weight; application of maximum snow action (“2”); application of maximum wind suction (“3”). The model predicted behavior provides that cable $C_{1}$ reaches 
the maximum value of internal tension under the snow action, while cable $C_{2}$ reaches the minimum value of tension; the cables length is modified under the load action, the initial geometry is bigger of lower in base of the direction of the load action. If the direction is gravitational the load bearing cables length increase, at contrary for the stabilizing cables. In this case, if wrongly designed, it is possible that the stabilizing cables curvature becomes inverse and the structure becomes instable. In Equations (1) and (2) the global cables stiffness $K_{c_{1}}$ and $K_{c 2}$ of the net is defined for the two order of cables; in Equations (4) and (5) $k_{1}$ and $k_{2}$ the single cables stiffness is defined depending on the cable area, sag, span, and finally on the cable material ( $E$ is the Young Module of the cable steel assumed equal to 165,000 Mpa).

$$
\begin{gathered}
K_{c_{1}}=\frac{k_{1}}{k_{1}+k_{2}} \\
K_{c_{2}}=\frac{k_{2}}{k_{1}+k_{2}} \\
k_{2}=\frac{E A_{1}}{l\left[1+\frac{8}{3}\left(\frac{\overline{f_{1}}}{l}\right)^{2}-\frac{32}{5}\left(\frac{\overline{f_{1}}}{l}\right)^{4}\right]} \\
l\left[1+\frac{8}{3}\left(\frac{E A_{2}}{l}\right)^{2}-\frac{32}{5}\left(\frac{\overline{f_{2}}}{l}\right)^{4}\right]
\end{gathered}
$$

The initial conditions are represented by the initial geometry, the load action considered and the material chosen in order to evaluate an initial value of cables stiffness, also a preliminary value of cables area $\left(A_{0,1}, A_{0,2}\right)$ and strain $\left(\epsilon_{0,1}, \epsilon_{0,2}\right)$ are fixed. This value will be iteratively modified. In Figure 3 the procedure 1 flow chart illustrates the first step of calculus. In the following the maximum snow action strain values are named $\epsilon_{2,1}$, $\epsilon_{2,2}$ and the maximum wind suction strain values are named $\epsilon_{3,1}, \epsilon_{3,2}$. The suffix " $i$ " used in the following equations indicates the generic load condition, $G_{p 1}$ is dead load, $G_{p 2}$ is permanent load, $S$ is the maximum snow action, $W$ is the maximum wind suction. Like a start point the ratio $\frac{k_{1}}{k_{2}}$ between the cables stiffness is assumed equal to $\Delta P$ defined in Equation (6).

$$
\Delta P=\left[\frac{G_{p 1}+G_{p 1}+|W|}{G_{p 1}+G_{p 1}+S}\right]=\frac{\text { Load Condition (3) }}{\text { Load Condition (2) }}
$$

The initial geometrical cables length $L_{0,1, \text { geom }}$ and $L_{0,2, \text { geom }}$ is evaluated using the approximated formulation reported in Equations (7) and (8), depending on cables initial sag and span.

$$
\begin{aligned}
& L_{0,1, \text { geom }}=L_{1} \cdot\left[1+\frac{8}{3} \cdot\left(\frac{f_{1}}{L_{1}}\right)^{2}-\frac{32}{5} \cdot\left(\frac{f_{1}}{L_{1}}\right)^{4}\right] \\
& L_{0,2, \text { geom }}=L_{2} \cdot\left[1+\frac{8}{3} \cdot\left(\frac{f_{2}}{L_{2}}\right)^{2}-\frac{32}{5} \cdot\left(\frac{f_{2}}{L_{2}}\right)^{4}\right]
\end{aligned}
$$

The initial cables internal traction $T_{0,1}$ and $T_{0,2}$ is evaluated as reported in Equations (9) and (10) assumed as the first forces of the mechanical problem; the initial horizontal traction $H_{0,1}$ equal to $H_{0,2}$ applied on the boundary structures is defined in Equation (11).

$$
T_{0,1}=H_{0,1} \sqrt{1+16\left(\frac{f_{1}}{L_{1}}\right)^{2}}
$$




$$
\begin{gathered}
T_{0,1}=H_{0,2} \sqrt{1+16\left(\frac{f_{2}}{L_{2}}\right)^{2}} \\
H_{0,1}=\frac{G_{p 1} L_{1}^{2}}{8 f_{1}} ; H_{0,2}=\frac{G_{p 1} L_{2}^{2}}{8 f_{2}}
\end{gathered}
$$

The initial balance between dead load and initial fixed is assumed as first condition. The load action is applied as an uniform distribution load (named equivalent load and in the following indicated as $P$ ) on the load bearing cable. In this particular case is assumed as a hypothesis that snow action is bigger than the wind suction. The reason is that there are snow loads value in the code for each altitude but there aren't wind loads data for Hyperbolic paraboloid shape; so the flat roof pressure coefficients is chosen as a start point. The wind load value evaluated with the flat roof pressure coefficients is general lower than the snow action for an altitude major than $200 \mathrm{~m}$, using the Eurocode. In order to preliminary design the cable net for the bigger load condition at first the snow action $(S)$ is evaluated (load condition 2). Using the procedure 1 illustrated in Figure 3, the geometry variation of the load bearing cable (in this case $C_{1}$ ) is evaluated in order to obtain the $H_{2,1}$ defined by Equation (12). It's important to note that $f_{2,1}$ and $T_{2,1}$ are the deformed sag and the cable $C_{1}$ traction with the snow action.

$$
H_{2,1}=\frac{T_{2,1}}{1+16\left(\frac{f_{2,1}}{L_{1}}\right)^{2}}
$$

In Equation (12) $T_{2,1}$, according to the Hook law is equal to $E \varepsilon A$, and $\epsilon$ and $A$ are the strain and the cable area that satisfy the balance in load condition 2. Replacing in Equations (2), (3), (13), (14) are defined.

$$
\begin{aligned}
& K_{C_{1}}=\left\{\frac{\left[\frac{E A_{1} f_{1}^{2}}{L_{1}^{4}}\right]}{\left[\frac{E A_{1} f_{1}^{2}}{L_{1}^{4}}\right]+\left[\frac{E\left(A_{2}\right) f_{2}^{2}}{L_{2}^{4}}\right]}\right\}=\left\{\frac{1}{\left.1+\frac{E\left(A_{2}\right) f_{2}^{2}}{L_{2}^{4}}\right]}\right\}=\left[\frac{1}{1+\frac{1}{\Delta P}}\right]
\end{aligned}
$$

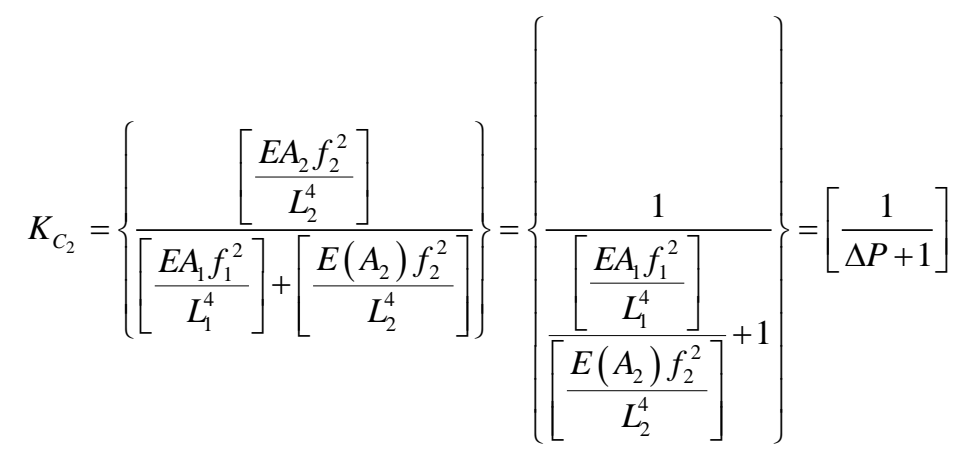

The load configuration $P_{2,1}$, (load configuration 2, cable 1) that corresponds to snow action, is evaluated according to the Equation (15) obtaining by the Equations (10) and (12) and dimensionless respect the cable area $A_{1}$. It is possible to define $P_{2,1}$ also according to Equation (16).

$$
\frac{P_{2,1}}{A_{1}}=\frac{8 \varepsilon_{2,1} f_{2,1}}{L_{1}^{2}} \Leftrightarrow P_{2,1}=\left(\frac{8 \varepsilon_{2,1} f_{2,1}}{L_{1}^{2}}\right) A_{1} \text { (iteration to } A_{1} \text { ) }
$$




$$
\overline{P_{2,1}}=\frac{8 H_{2,1} f_{2,1}}{L_{1}^{2}}
$$

The relation that connect the "2" load condition and the "0" load condition is reported in Equations (17) and (18). The load balance reported in Equation (18) is obtained with an iteration of $A_{1}$.

$$
\begin{gathered}
P_{0,1}=\left[\left(\frac{P_{0,1}}{A_{1}}\right) A_{1}\right]-\left[\frac{\left(G_{p 1}+G_{p 1}\right)}{\left(\frac{1}{K_{c_{1}}}\right)}\right]=\left[\left(\frac{8 \varepsilon_{0,1} f_{0,1}}{L_{1}^{2}}\right) A_{1}\right]-\left[\frac{\left(G_{p 1}+G_{p 1}\right)}{\left.\left(\frac{1}{K_{c_{1}}}\right)\right]}\right] \\
\Delta=\left\{P_{0,1}+\left[\frac{\left(G_{p 1}+G_{p 1}+S\right)}{\left(\frac{1}{K_{c_{1}}}\right)}-\frac{\left(G_{p 1}+G_{p 1}\right)}{\left(\frac{1}{K_{c_{1}}}\right)}\right]\right\}-P_{2,1} \\
\left.=\left\{\left[\left(\frac{8 \varepsilon_{0,1} f_{0,1}}{L_{1}^{2}}\right) A_{1}\right]-\left[\frac{(S)}{\left.\left(\frac{1}{K_{c_{1}}}\right)\right]}\right]\right\}-\left[\frac{8 \varepsilon_{2,1} f_{2,1} A_{1}}{L_{1}^{2}}\right]=0 \quad \text { (iteration to } A_{1} \text {, fixed } \varepsilon_{0,1} \text { and } f_{0,1}\right)
\end{gathered}
$$

With the same procedure $A_{2}$ is fixed updating the value of $A_{1}$. In conclusion, fixed an initial condition of traction the cables areas are defined according to the initial geometry wanted. In Figures 2-4 the flow charts of the procedure described are shown in order to summarize step by step the numerical proceedings. To validate the numerical procedure a comparison of the cables structural response with a Finite element method analyses (in the following FE) is done; in Figure 5(a) and Figure 5(b) the vertical displacements of the middle node is plotted for different cables areas but with same load applied (in this case equal to $2.2 \mathrm{kN} / \mathrm{m}$ gravitational and uniform load on the load bearing cable). The load bearing cable sag and span are equal to 4.44 and $80 \mathrm{~m}$, the stabilizing cables sag and span are equal to 8.89 and $80 \mathrm{~m}$; in this case the ratio $\frac{A_{1}}{A_{2}}$ is assumed equal to 2 . The mean value of the percentage error is equal to $12 \%$. This value appear acceptable if the approximation is considered. In the following the numerical procedure described in this section will be named NPPD (Numerical Procedure of Preliminary Design) (Elashkar I., Novak M., 1983; Lewis W. J., 2004; Majowiecki M., 2004) [8]-[10].

\subsection{The Analyzed Geometric Sample}

In order to estimate a set of optimal geometry with the minimum cables areas and displacements (for equal forces transmitted to the support structure) [11]-[15], a geometric parameterization taking into account the following parameters was carried out (Figure 6):

- $\gamma$, the relationship between the cable sags, $\left(f_{2} / f_{1}\right)$; 8 different values of $\gamma$, respectively equal to $0.43,0: 50,0.70$, $1.00,1.501 .80,2.00,2.33$ were taken into account;

- $\rho$, the relationship between the roof height and the maximum span length $\left(H / L_{\max }\right) ; 6$ different values of $\rho$, respectively equal to $1 / 3,1 / 4,1 / 5,1 / 6,1 / 8,1 / 10$ were taken into consideration.

- $\alpha$, the relationship between the span length $\left(L_{1} / L_{2}\right)$; 4 different values of $\alpha$, respectively equal to 1.50 (rectangular plan shape), 1.00 (square plan shape), 0.50 (rectangular plan shape), and variable, for structures with a circular plan shape were taken into account.

With the previously numerical procedure, 1008 different geometrical combinations were analyzed; only some configurations meet the optimization criteria pre-fixed in the hypotheses; in particular:

- Cables net with $L_{1}<L_{2}$ show better performance with lower values of forces transmitted to the supports.

- Cables net with $\gamma>1$ gives higher displacements but lower cables areas and therefore a lower structural 
weight.

- “Optimal” values of $\gamma$, both as regards stress and structural weight optimization, are in the range between 1.5 and 2.5.

- Low values of $\rho$ give higher cables areas and therefore a higher structural weight; however, they gives "optimal" stresses with respect to cables net with high value of $\rho$.

- The ratio between the obtained displacements with lower span lengths and those obtained with higher spans are lower compared to a direct proportionality.

Totally one thousand geometries are investigate in order to compare the structural response and to choose an optimal sample to test in wind tunnel. On the basis of these preliminary results, a representative geometric sample to be tested in the wind tunnel was chosen; in Table 1 the geometrical sizes of the full scale cable nets are listed for each plan shape. This phase of the work has produced a preliminary design numerical procedure with which it is possible to identify the sample to test in wind tunnel. A model scale equal to 1:100 is chosen to construct the models.

\section{Numerical Procedure to Generate Cables Net Fe Models}

Before wind tunnel test, tridimensional FE analyses are performed in order to simulate examples of full scale structures. The weight of the cables nets and their structural response under the snow action are studied to verify that the geometries chosen give a high structural response. In order to provide the geometric input for FEM models

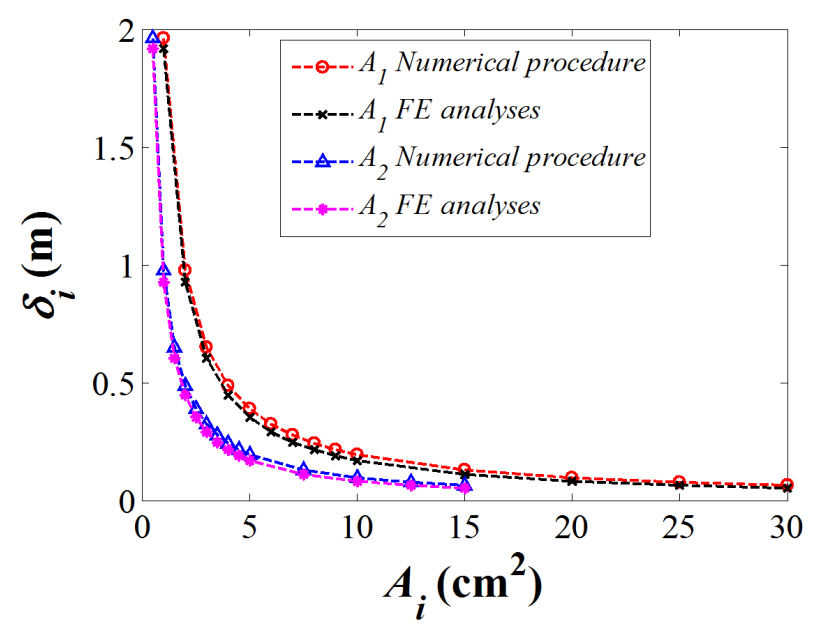

(a)

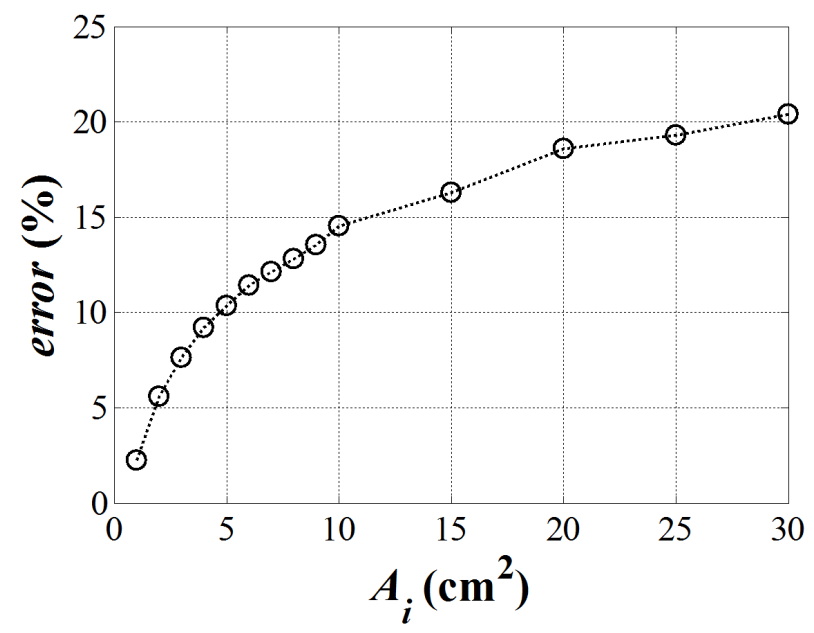

(b)

Figure 5. (a) Comparison between FEM analysis and procedure of preliminary design and (b) percentage error. 


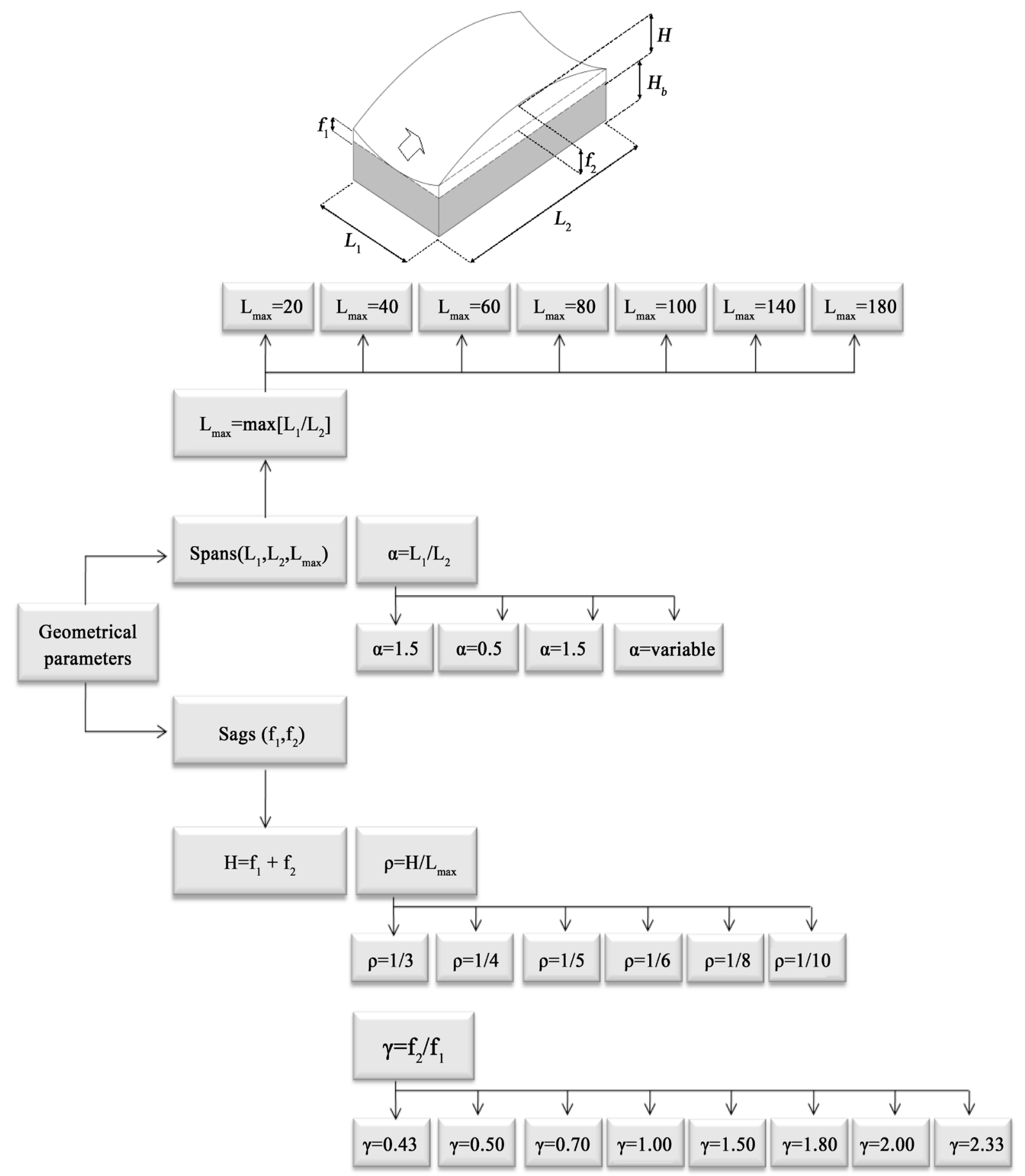

Figure 6. Geometrical configurations investigated with the numerical procedure for cables net preliminary design.

with Hyperbolic Paraboloid shapes a numerical procedure has been implemented. At first, a step-by-step procedure allows to describe the two-dimensional domain and the three-dimensional domain firstly by choosing a reference system (Cartesian or polar). It is possible to compute the two-dimensional domain in two different ways; in both cases, the procedure allows to choose among four different conventional shapes: circle, ellipsis, rectangle and polygon. In the first case, the user must enter the geometric parameters of the shapes, for example, the radius or the side's length. The second case allows calculating the curve function by choosing through an equations system obtained by setting some points coordinates on the domain. An additional option allows to directly 
Table 1. Geometrical sample.

\begin{tabular}{|c|c|c|c|c|c|c|c|c|c|}
\hline \multicolumn{10}{|c|}{ Square plan model } \\
\hline model & $\alpha$ & $\rho$ & $\gamma$ & $f_{1}[\mathrm{~m}]$ & $f_{2}[\mathrm{~m}]$ & $L_{1}[\mathrm{~m}]$ & $L_{2}[\mathrm{~m}]$ & $H[\mathrm{~m}]$ & $H_{b}[\mathrm{~m}]$ \\
\hline p.1 & 1 & $1 / 10 L_{\max }$ & 2 & 2.67 & 5.33 & 80.00 & 80.00 & $8.00\left(1 / 10 L_{\max }\right)$ & $13.33\left(1 / 6 L_{\max }\right)$ \\
\hline p.2 & 1 & $1 / 10 L_{\max }$ & 2 & 2.67 & 5.33 & 80.00 & 80.00 & $8.00\left(1 / 10 L_{\max }\right)$ & $26.66\left(1 / 3 L_{\max }\right)$ \\
\hline p.7 & 1 & $1 / 6 L_{\max }$ & 2 & 4.44 & 8.89 & 80.00 & 80.00 & $13.33\left(1 / 6 L_{\max }\right)$ & $13.33\left(1 / 6 L_{\max }\right)$ \\
\hline p.8 & 1 & $1 / 6 L_{\max }$ & 2 & 4.44 & 8.89 & 80.00 & 80.00 & $13.33\left(1 / 6 L_{\max }\right)$ & $26.66\left(1 / 3 L_{\max }\right)$ \\
\hline \multicolumn{10}{|c|}{ Rectangular plan model } \\
\hline & $\alpha$ & $\rho$ & $\gamma$ & $f_{1}[\mathrm{~m}]$ & $f_{2}[\mathrm{~m}]$ & $L_{1}[\mathrm{~m}]$ & $L_{2}[\mathrm{~m}]$ & $H[\mathrm{~m}]$ & $H_{b}[\mathrm{~m}]$ \\
\hline p.3 & 0.5 & $1 / 6 L_{\max }$ & 2 & 4.44 & 8.89 & 40.00 & 80.00 & $13.33\left(1 / 6 L_{\max }\right)$ & $13.33\left(1 / 6 L_{\max }\right)$ \\
\hline p.4 & 0.5 & $1 / 6 L_{\max }$ & 2 & 4.44 & 8.89 & 40.00 & 80.00 & $13.33\left(1 / 6 L_{\max }\right)$ & $26.66\left(1 / 3 L_{\max }\right)$ \\
\hline p.5 & 0.5 & $1 / 10 L_{\max }$ & 2 & 2.67 & 5.33 & 40.00 & 80.00 & $8.00\left(1 / 10 L_{\max }\right)$ & $13.33\left(1 / 6 L_{\max }\right)$ \\
\hline p.6 & 0.5 & $1 / 10 L_{\max }$ & 2 & 2.67 & 5.33 & 40.00 & 80.00 & $8.00\left(1 / 10 L_{\max }\right)$ & $26.66\left(1 / 3 L_{\max }\right)$ \\
\hline \multicolumn{10}{|c|}{ Circular plan model } \\
\hline & $\alpha$ & $\rho$ & $\gamma$ & $f_{1}[\mathrm{~m}]$ & $f_{2}[\mathrm{~m}]$ & $D_{1}[\mathrm{~m}]$ & $D_{2}[\mathrm{~m}]$ & $H[\mathrm{~m}]$ & $H_{b}[\mathrm{~m}]$ \\
\hline p.9 & 1 & $1 / 6 L_{\max }$ & 2 & 4.44 & 8.89 & 80.00 & 80.00 & $13.33\left(1 / 6 L_{\max }\right)$ & $13.33\left(1 / 6 L_{\max }\right)$ \\
\hline p.10 & 1 & $1 / 6 L_{\max }$ & 2 & 4.44 & 8.89 & 80.00 & 80.00 & $13.33\left(1 / 6 L_{\max }\right)$ & $26.66\left(1 / 3 L_{\max }\right)$ \\
\hline p.11 & 1 & $1 / 10 L_{\max }$ & 2 & 2.67 & 5.33 & 80.00 & 80.00 & $8.00\left(1 / 10 L_{\max }\right)$ & $13.33\left(1 / 6 L_{\max }\right)$ \\
\hline p.12 & 1 & $1 / 10 L_{\max }$ & 2 & 2.67 & 5.33 & 80.00 & 80.00 & $8.00\left(1 / 10 L_{\max }\right)$ & $26.66\left(1 / 3 L_{\max }\right)$ \\
\hline \multicolumn{10}{|c|}{ Elliptical plan model } \\
\hline & $\alpha$ & $\rho$ & $\gamma$ & $f_{1}[\mathrm{~m}]$ & $f_{2}[\mathrm{~m}]$ & $L_{1}[\mathrm{~m}]$ & $L_{2}[\mathrm{~m}]$ & $H[\mathrm{~m}]$ & $H_{b}[\mathrm{~m}]$ \\
\hline p.14 & 0.5 & $1 / 10 L_{\max }$ & 2 & 2.67 & 5.33 & 80.00 & 80.00 & $8.00\left(1 / 10 L_{\max }\right)$ & $13.33\left(1 / 6 L_{\max }\right)$ \\
\hline p.15 & 0.5 & $1 / 10 L_{\max }$ & 2 & 2.67 & 5.33 & 80.00 & 80.00 & $8.00\left(1 / 10 L_{\max }\right)$ & $26.66\left(1 / 3 L_{\max }\right)$ \\
\hline p.16 & 0.5 & $1 / 6 L_{\max }$ & 2 & 4.44 & 8.89 & 80.00 & 80.00 & $13.33\left(1 / 6 L_{\max }\right)$ & $13.33\left(1 / 6 L_{\max }\right)$ \\
\hline p.17 & 0.5 & $1 / 6 L_{\max }$ & 2 & 4.44 & 8.89 & 80.00 & 80.00 & $13.33\left(1 / 6 L_{\max }\right)$ & $26.66\left(1 / 3 L_{\max }\right)$ \\
\hline
\end{tabular}

importing a.dxf (Drawing Interchange Format) file that describes the two-dimensional domain or, in the case of complex and irregular domains, it is possible to directly import the coordinates of the shape vertices. After setting the two-dimensional domain, the three-dimensional one can be set by choosing among seven prefixed shapes (sphere, ellipsoid, flat, hyperbolic paraboloid, elliptic paraboloid, a one slope hyperboloid and a two slopes hyperboloid). Also in this case it is possible to directly insert geometric parameters and surfaces coefficients, or compute their functions through an equations system obtained by setting some points coordinates on the surface. The next step concern the insertion of the cables spacing in the two directions, $X$ and $Y$ in the case of Cartesian coordinates, or meridians and parallels in the case of polar coordinates. In the case of Cartesian system, the procedure computes the equation of each line that describes the cable, and then intersects lines with the 2D domain generating a set of nodes and computing the respective coordinates on the plane, $p_{i}\left(x_{p}, y_{p}\right)$. In the next step the procedure projects the evaluated nodes on the spatial surface, identifying the third coordinate $z_{p}$.

In the exporting phase, 3 different files can be saved: a.txt (text file) for input that contains the number of nodes and their coordinates; a.dxf with the cables net vector model; and a file that contains the functions equations of the created curves and surfaces. Thanks to this procedure, FEM model for nonlinear dynamic analyses can easily be generated. Figure 7(a) shows the intersection between the 2D domain and the 3D domain, while Figure 7(b) shows the simplified flow chart of the numerical procedure. Finally, in Figure 7(c) an example of hyperbolic paraboloid mesh with square plan shape generated is reported. Static and modal FE analyses are performed using the numerical models tested. Using the .dxf files generated the wind tunnel models are constructed made of wood. In the following the numerical procedure described in this section will be named Numerical Procedure to Generate Finite Element Models, in the following NPGFM [16]-[18].

\section{Wind Tunnel Test}

\subsection{Setup}

The wind tunnel is a tool used in aerodynamic, aeroelastic and fluid mechanics research to study the effects of 


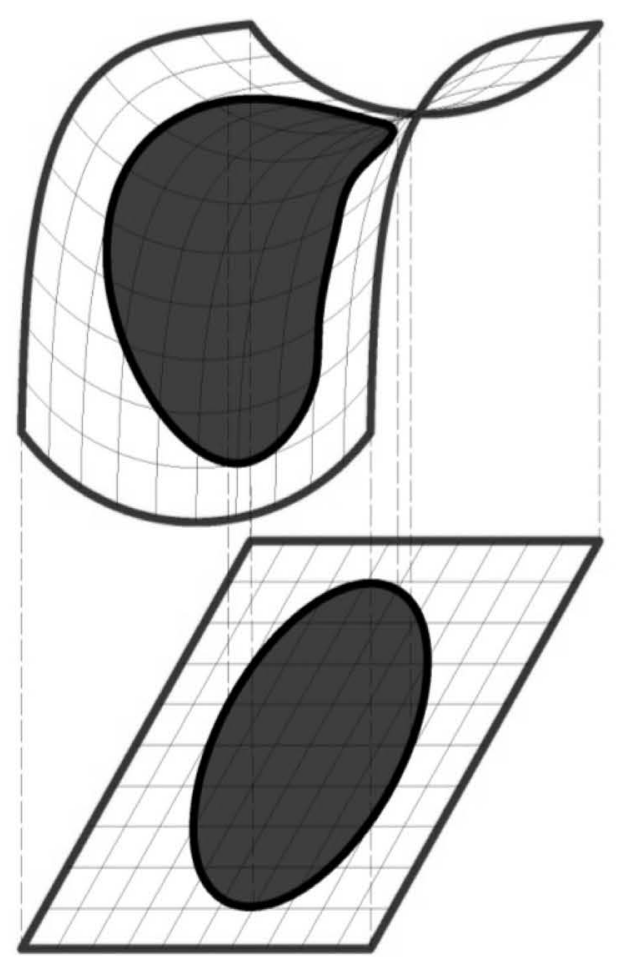

(a)

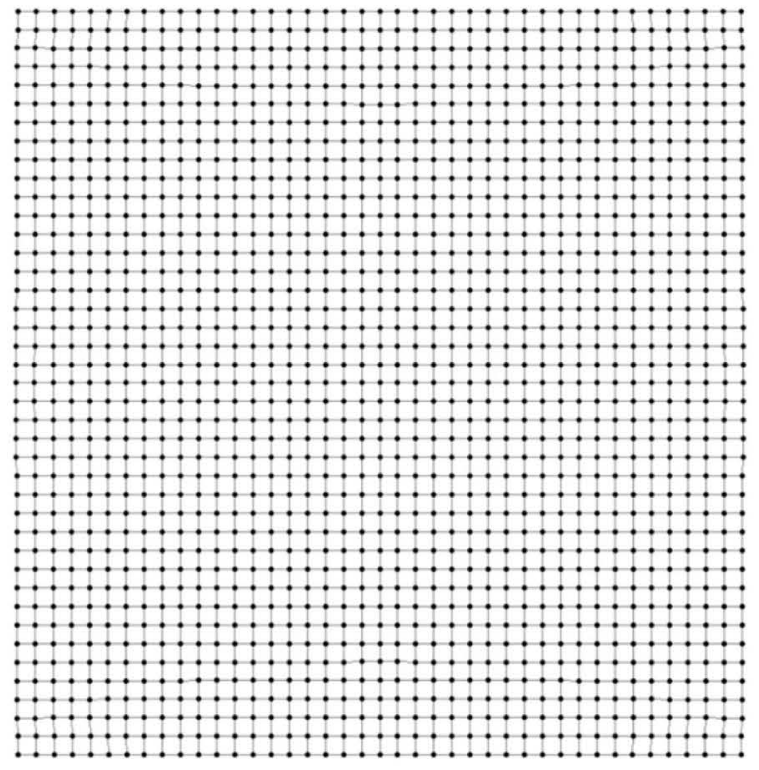

(c)

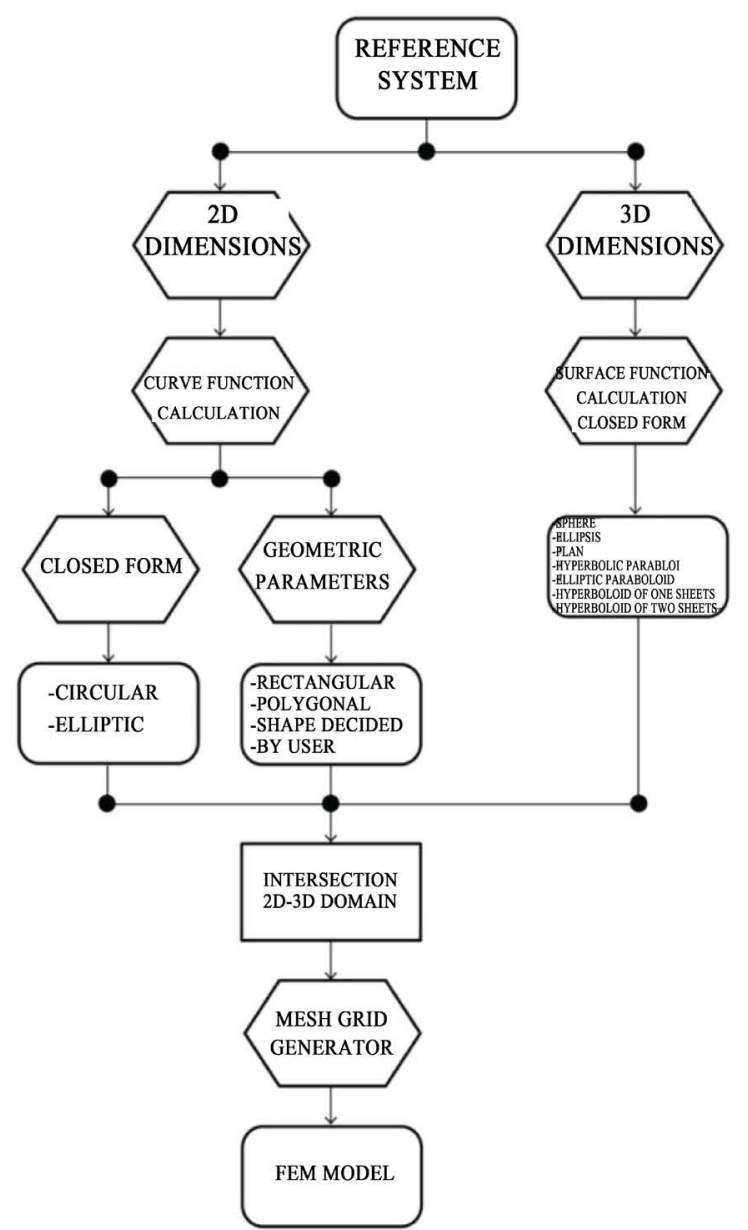

(b)

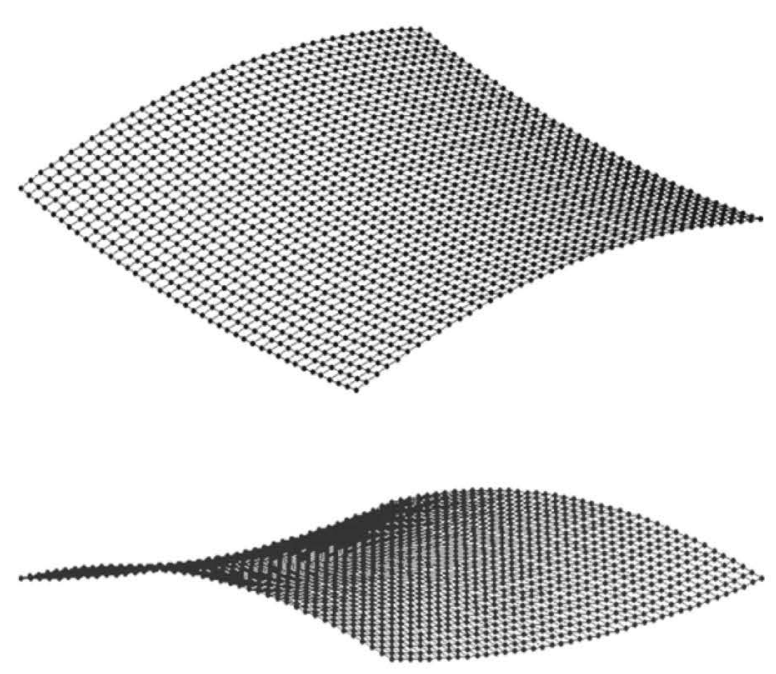

Figure 7. (a) Intersection between 2D and 3D domain; (b) Tensile mesh generator flow chart; (c) Hyperbolic paraboloid mesh with square plan shape. 
air moving past solid objects rigid and flexible. A wind tunnel consists of a tubular passage with a particular geometry where the object to test is in the middle of the test chamber. Air is made to move past the object by a powerful fan system or other means. The wind tunnel model is instrumented with suitable sensors to measure aerodynamic forces, pressure distribution, or other aerodynamic-related characteristics. In this experience the models are rigid and the goals were to acquire pressure coefficients [19]-[25].

The CRIACIV (Inter-University Research Center for Building Aerodynamics and Wind Engineering) wind tunnel located in Prato (Italy) is used to perform aerodynamic experiments. A layout of the wind tunnel is illustrated in Figure 8. The choice of the turbulence intensity and so of the wind tunnel speed profile is important, too. For this research a more general possible condition was necessary. A medium urban profile is chosen for the boundary layer. In Figure 9(a) and Figure 9(b) the boundary layer development artificial roughness (wood panels) (a), spires (b) are shown and in Figure 9(c) and Figure 9(d) the speed and turbulence profile is reported. The mean value of the wind tunnel speed at the roof level is between 16 and $20 \mathrm{~m} / \mathrm{s}$, the turbulence at the same level is between $12 \%$ and 15\% (Simiu E., Scanlan, R. H., 1986).

The test models are made in wood and their geometrical scale is fixed equal to 1:100; the reason of this choice is to have big models and so easy to construct but with a not much high blockage coefficients. The constructions follows the geometry designed using the numerical procedure described in Section 3. Test models pictures are shown in Figure 10, respectively with a square plan (a), rectangular plan (b), circular plan (c) and elliptical plan (d). The geometry of the wind tunnel models is described in Table 1. Each model was instrumented (Figure 11(a) and Figure 11(b)) varying from 175 to 211 pressure taps distributed on the roof like shown in Figure 11(c). Each pressure tap was connected to a pressure transducers with a pneumatic connection made of Teflon tubes with $1.3 \mathrm{~mm}$ internal diameters (Figure 11(a) and Figure 11(b)). Data for 16 different wind angles were acquired at a frequency of $252 \mathrm{~Hz}$ and for 30 seconds obtaining, for each pressure tap, a pressure time history of 7504 values.

The ratio between the models size and the test chamber section size is very important $(\varphi)$. If this ratio is big ( $2 \%$ or $3 \%$ ) the blockage effects increases pressure coefficients and correction factors are necessary. In this case the blockage effect is not negligible and so a blocking coefficient $\beta$ was considered. According to Equation (19) $\varphi$ is evaluated and the correction factor $\beta$ is estimate. $A_{\text {tot }}$ is the wind tunnel test section area and $A_{\text {lateral }}$ is the model section area.

$$
\varphi=\left(\frac{A_{\text {tot }}-A_{\text {lateral }}}{A_{\text {tot }}}\right)^{2}
$$

In Table 2 blockage values and the correction coefficients for each tested model are reported. They are in a range between $1.5 \%$ and $7.7 \%$ with peaks for geometries p.2, p.4, p.6, p.8, p.15 and p.17 (highest model). The correction values for the pressure coefficients vary from a minimum of $2 \%$ to a maximum of $4 \%$.

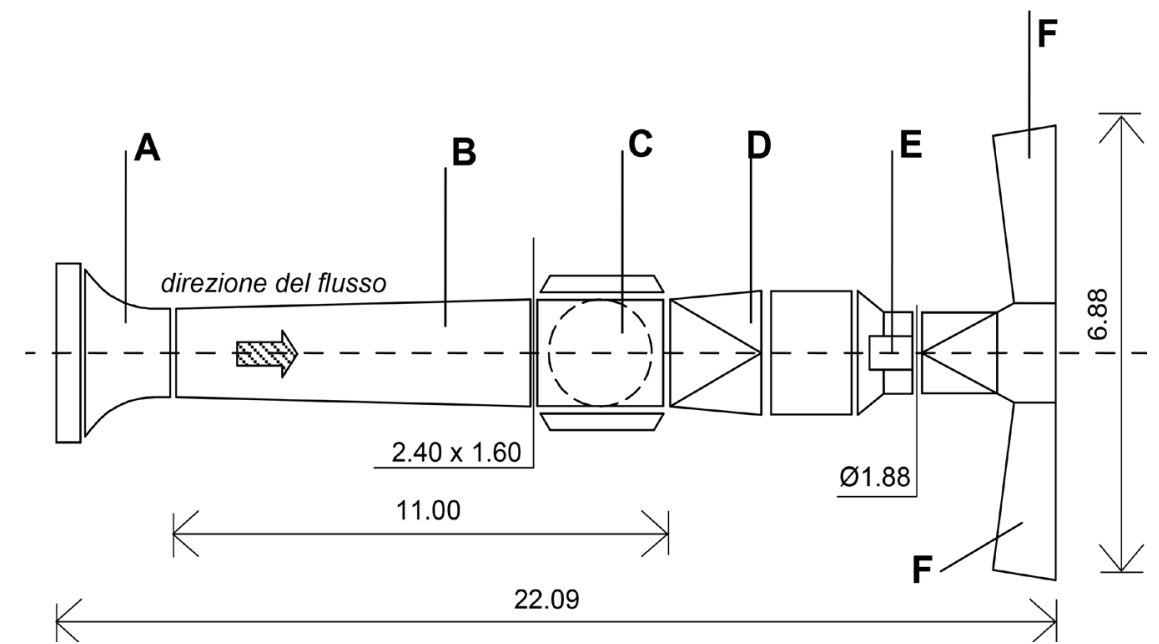

Figure 8. CRIACIV wind tunnel image (a), elements (b): (A) entrance; (B) boundary layer development zone; (C) test chamber (section size $2.40 \times 1.60 \mathrm{~m})$; (D) connection zone; (E) engine $(160 \mathrm{~kW})$; (F) diffusion T-shape. 


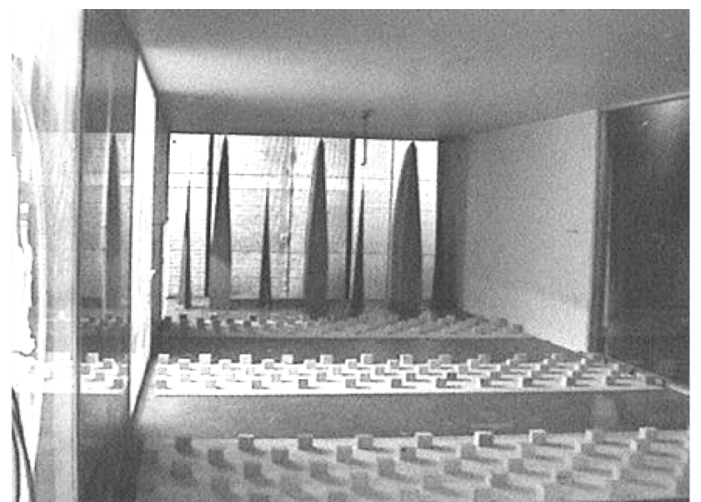

(a)

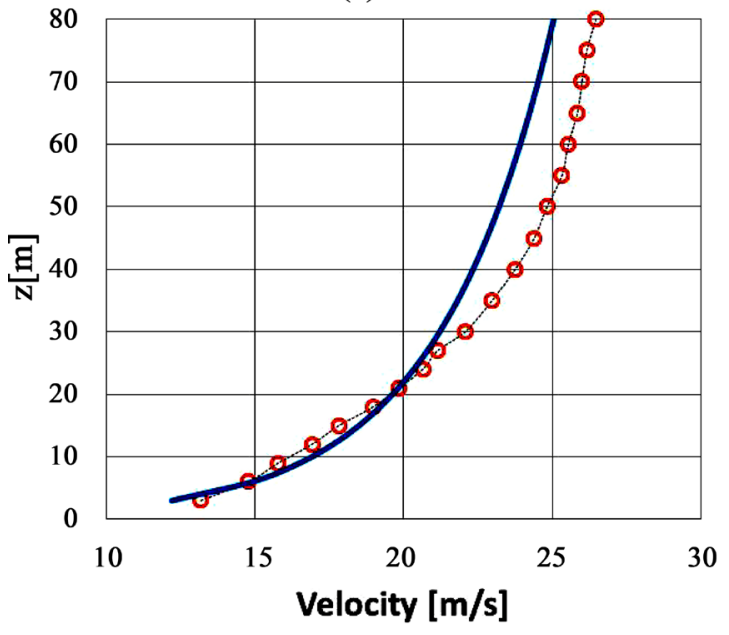

- Vmedia Appr. logaritmica

(c)

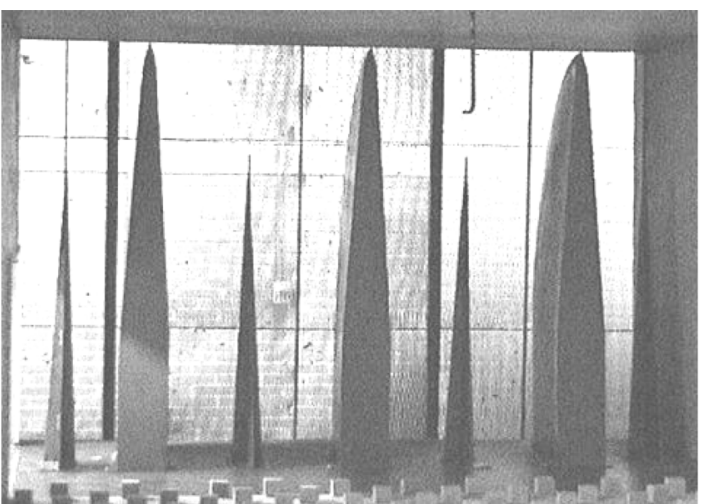

(b)

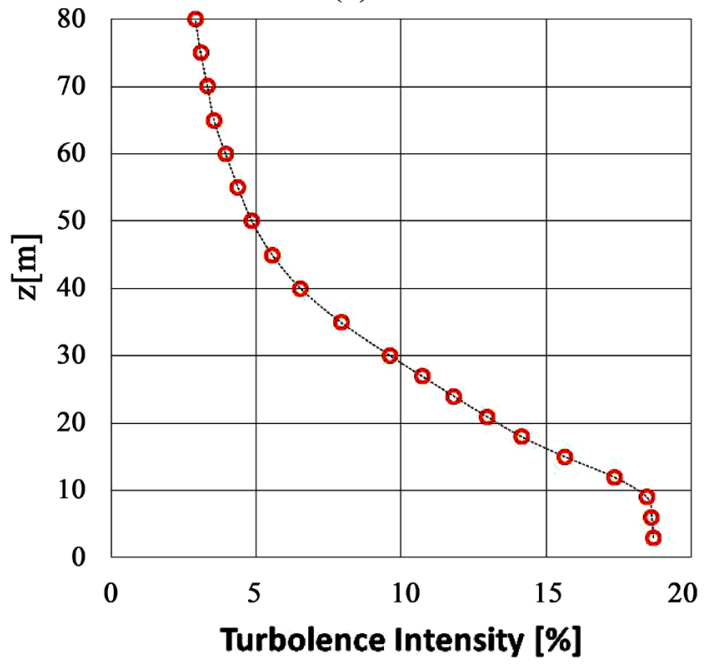

(d)

Figure 9. Boundary layer development artificial roughness (wood panels) (a), spires (b); (c) velocity and (d) turbulence profiles.

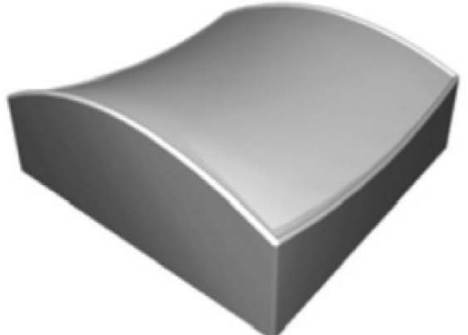

(a)

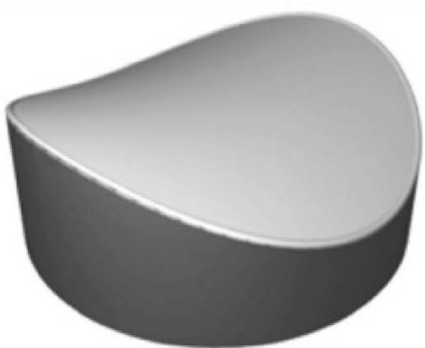

(c)

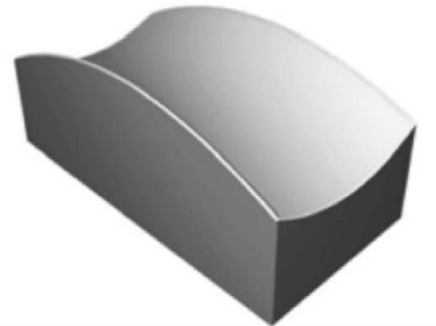

(b)

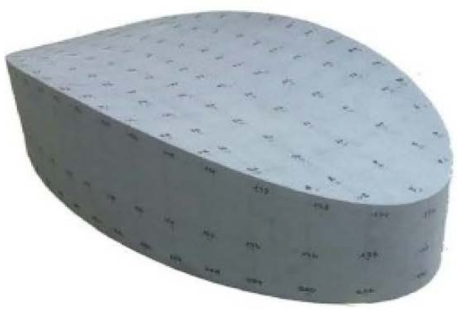

(d)

Figure 10. Hyperbolic parabolic roofs: (a) square plane; (b) rectangular plane; (c) circular plane; (d) ellipltical plane [13]. 


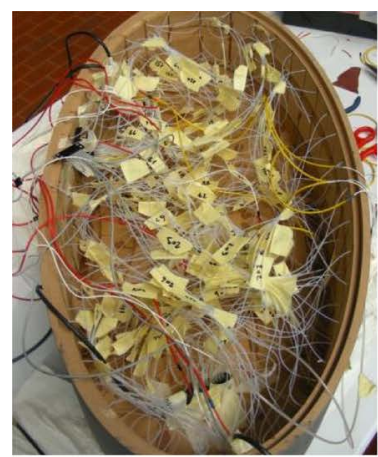

(a)

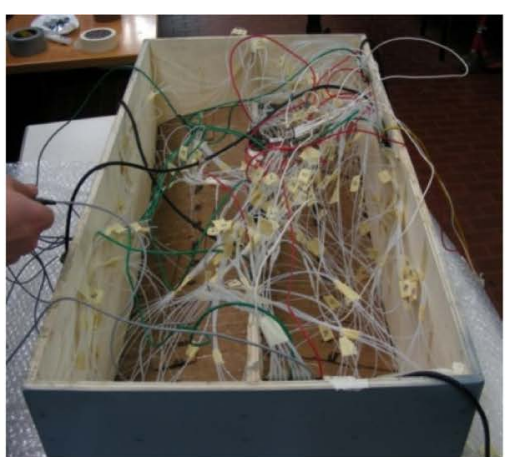

(b)

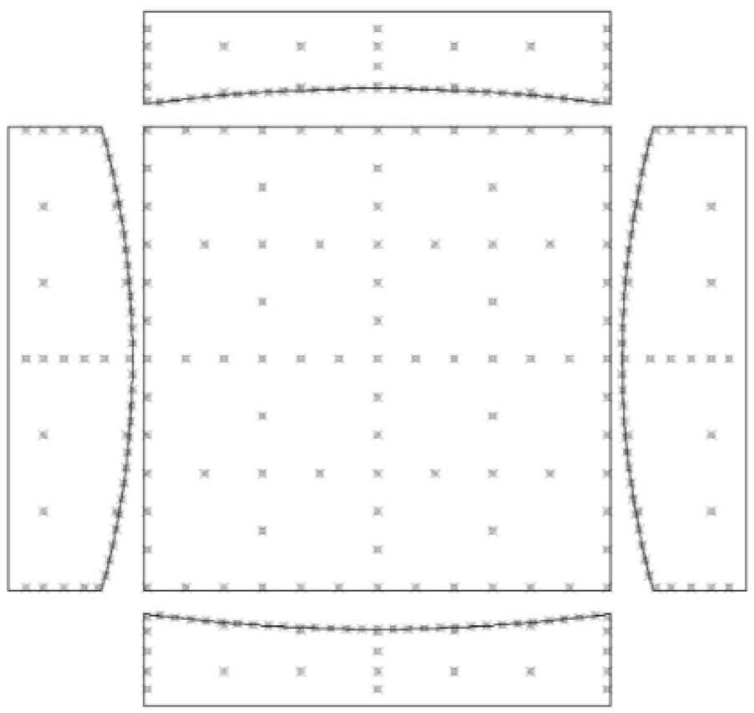

(c)

Figure 11. Wind tunnel acquisition data, (a) and (b) model instrumentation, (c) example of pressure taps distribution.

Table 2. Blockage values.

\begin{tabular}{|c|c|c|c|c|c|}
\hline & \multirow{3}{*}{ Shape } & \multicolumn{4}{|c|}{ Side2 } \\
\hline & & & & & \\
\hline & & Side 1 & Side 2 & Side 1 & Side 2 \\
\hline p.1 & square & 0.029 & 0.044 & 0.98 & 0.98 \\
\hline p. 2 & square & 0.057 & 0.072 & 0.97 & 0.96 \\
\hline p.3 & rectangular & 0.015 & 0.049 & 0.99 & 0.97 \\
\hline p.4 & rectangular & 0.029 & 0.077 & 0.98 & 0.96 \\
\hline p.5 & rectangular & 0.015 & 0.044 & 0.99 & 0.98 \\
\hline p.6 & rectangular & 0.028 & 0.072 & 0.99 & 0.96 \\
\hline p.7 & square & 0.031 & 0.049 & 0.98 & 0.97 \\
\hline p.8 & square & 0.058 & 0.077 & 0.97 & 0.96 \\
\hline p.14 & elliptical & 0.029 & 0.044 & 0.98 & 0.98 \\
\hline p.15 & elliptical & 0.057 & 0.072 & 0.97 & 0.96 \\
\hline p.16 & elliptical & 0.031 & 0.049 & 0.98 & 0.97 \\
\hline p.17 & elliptical & 0.058 & 0.077 & 0.97 & 0.96 \\
\hline
\end{tabular}




\subsection{Data Processing}

Experimental data consist in pressure expressed in $\mathrm{mmH}_{2} \mathrm{O}$ deriving by the transducers acquisition; a numerical procedure is needed in order to obtain a double goal: at first pressure in Pascal and pressure coefficients located on the roof and at second, forces time history to use in FEM analysis. For this reason a numerical procedure have to transform data into pressures, then evaluate the pressure coefficients, then estimate the maximum, minimum and mean values of these coefficients, and finally transform the pressure coefficients into forces to be applied on the FEM model. The first phase concerns the graphic aspect, with the preparation of the geometry of the pressure coefficients map; the second stage involves the implementation of the subroutine for the evaluation of the pressure coefficients and the third phase provides the forces calculation.

\subsubsection{Pressure Coefficients Maps}

In order to obtain pressure coefficients maps to describe the pressure distribution on the roof and sides of the model, the surface was discretized in polygons surrounding each pressure taps. The Thiessen (Voronoi) polygons theory is adopted. It consists to define individual areas of influence around each of a set of points. Thiessen polygons are polygons whose boundaries define the area that is closest to each point relative to all other points.

They are mathematically defined by the perpendicular bisectors of the lines between all points. The process goes through several steps: collects the points from a point layer (vertices if the source is a polyline or polygon layer), clean duplicate points, generates Convex Hull, creates a TIN structure, generates perpendicular bisectors for each tin edge, builds the Thiessen polygons and, finally, clips the Thiessen polygons feature class with the convex hull. A specific numerical procedure is programmed for this section. An example of Thiessen polygons distribution is illustrated in Figure 12. It is possible to note that if the pressure taps distribution is not geometrically regular with a structured grid, the shape of the Thiessen polygon is irregular. For each polygon one value of pressure coefficient is associated [19]-[25].

\subsubsection{Evaluation of Pressure Coefficients}

During wind tunnel tests the model is fixed in the test chamber, in Figure 13(a) a picture of a model during the test is shown. The tubes connected to the transduces sent an input to the computer that measure the pressure variation in $\mathrm{mm} \mathrm{H}_{2} \mathrm{O}$. The experimental data needs to be transformed in pressure coefficients. Pressure coefficients $\left(C_{p}\right)$, is a dimensionless number which describes the relative pressures throughout a flow field in fluid

\begin{tabular}{|c|c|c|c|c|c|c|}
\hline 9 & \multirow{3}{*}{13} & \multirow{3}{*}{10} & 19 & \multirow[b]{2}{*}{23} & \multirow{3}{*}{26} & 18 \\
\hline 8 & & & 20 & & & 17 \\
\hline 7 & & & 21 & 24 & & 16 \\
\hline 6 & 12 & 11 & 22 & 24 & 25 & 15 \\
\hline
\end{tabular}
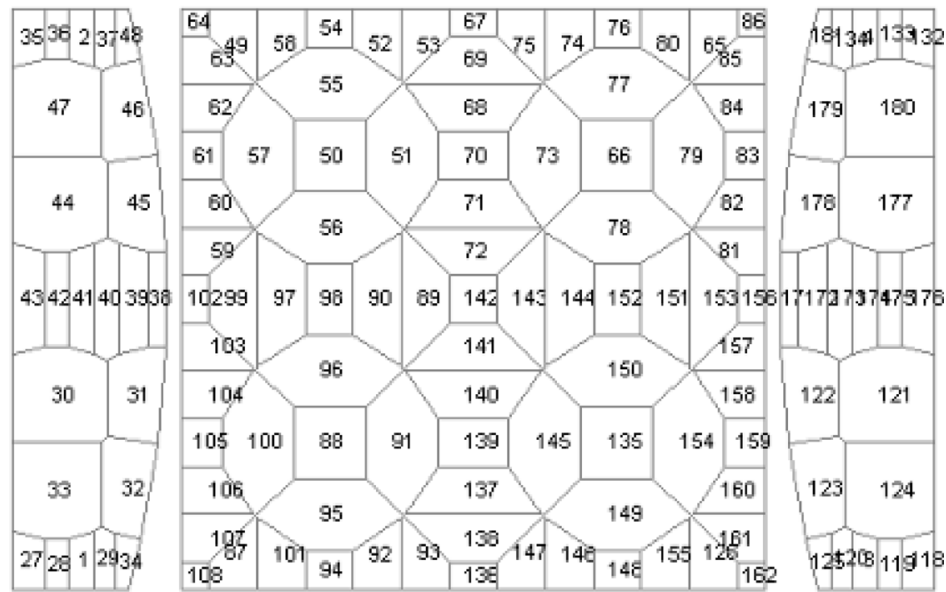

\begin{tabular}{|c|c|c|c|c|c|c|}
\hline 110 & 116 & 115 & & 168 & 169 & 12 \\
\hline 111 & \multirow{3}{*}{117} & \multirow{3}{*}{114} & 165 & & \multirow{3}{*}{170} & 12 \\
\hline 11 & & & 184 & \multirow{2}{*}{167} & & 130 \\
\hline 11 & & & 163 & & & 131 \\
\hline
\end{tabular}

Figure 12. An example of Thiessen polygons distribution. 


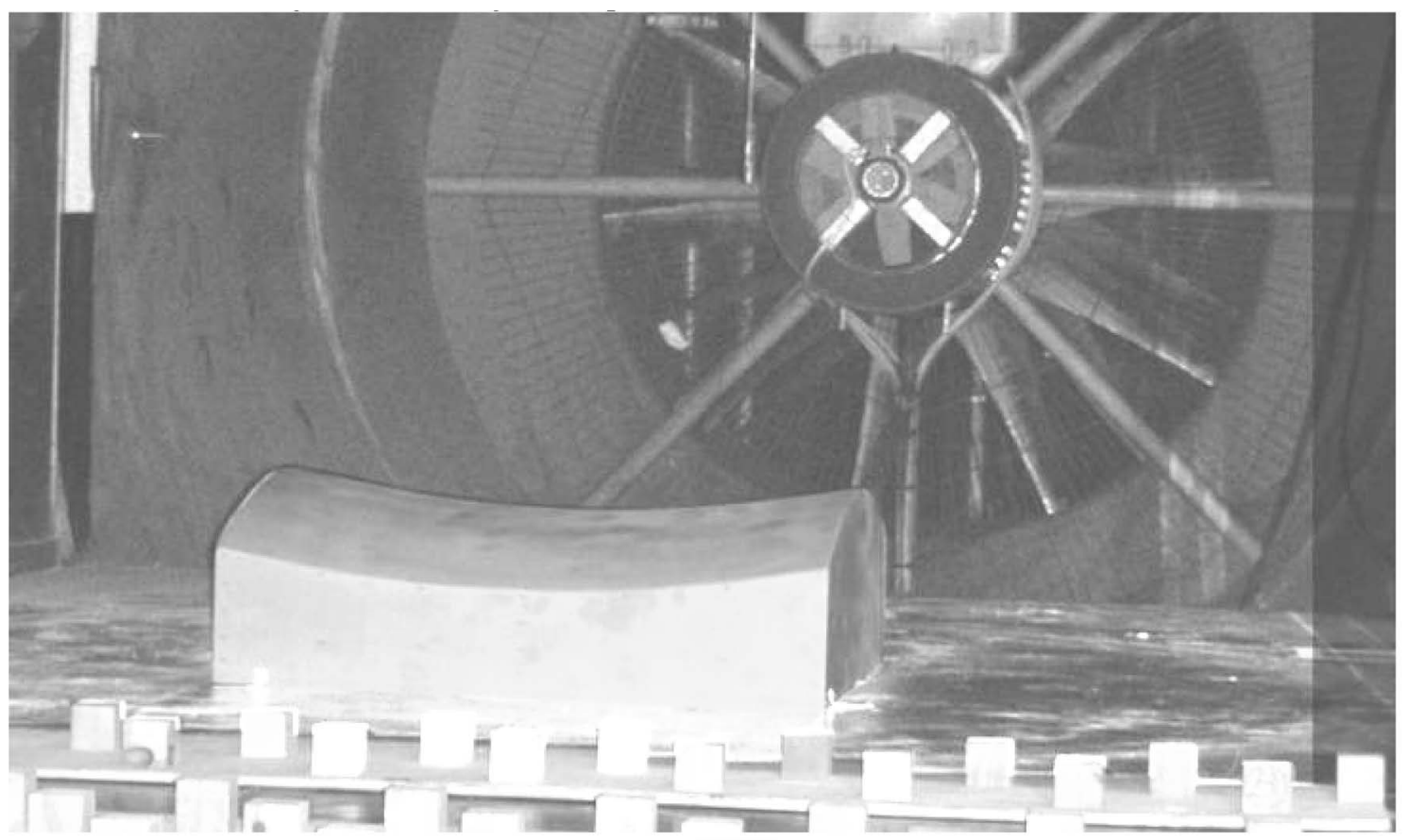

(a)

$C_{p}$ medi di progetto [file: dati-0000]

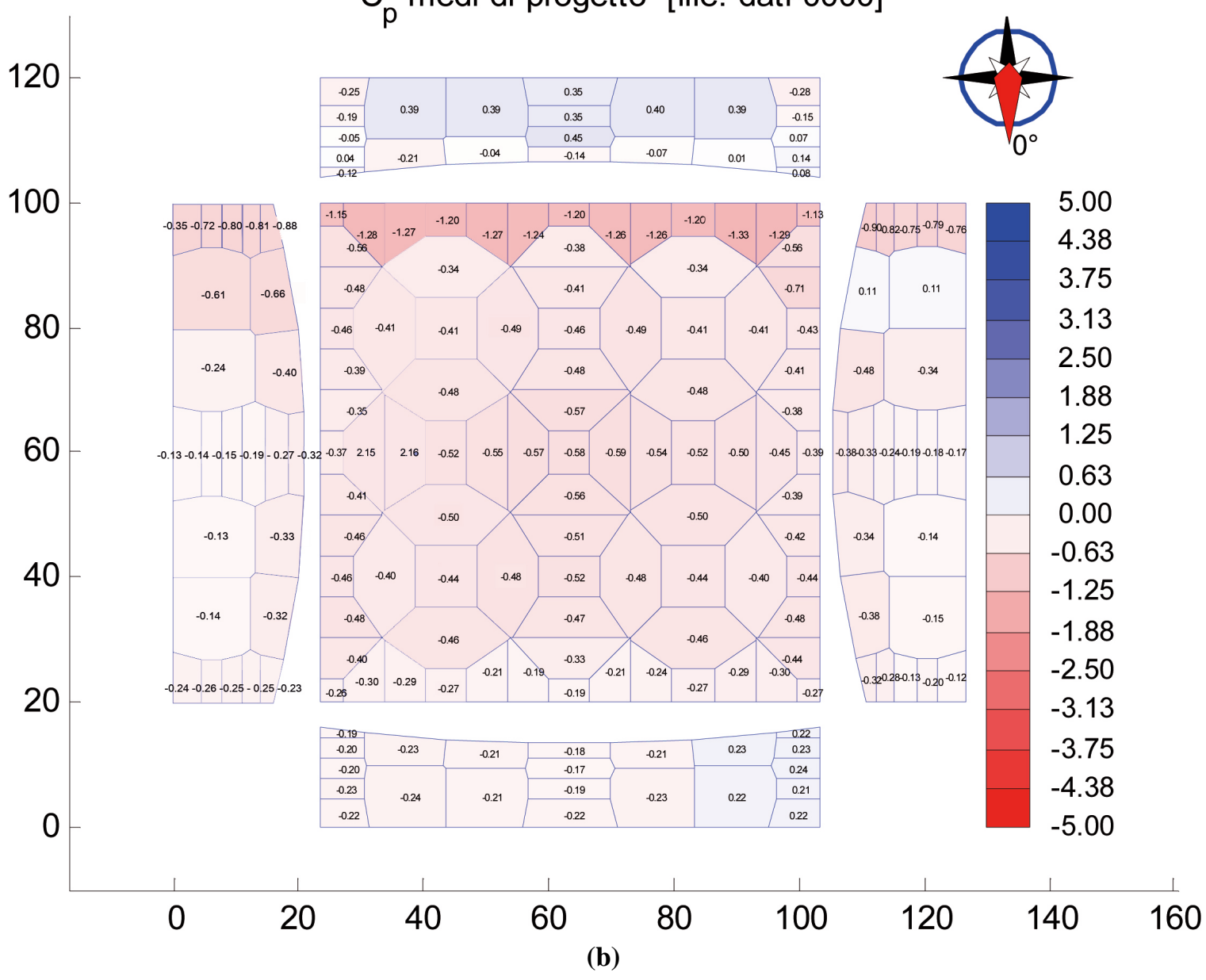




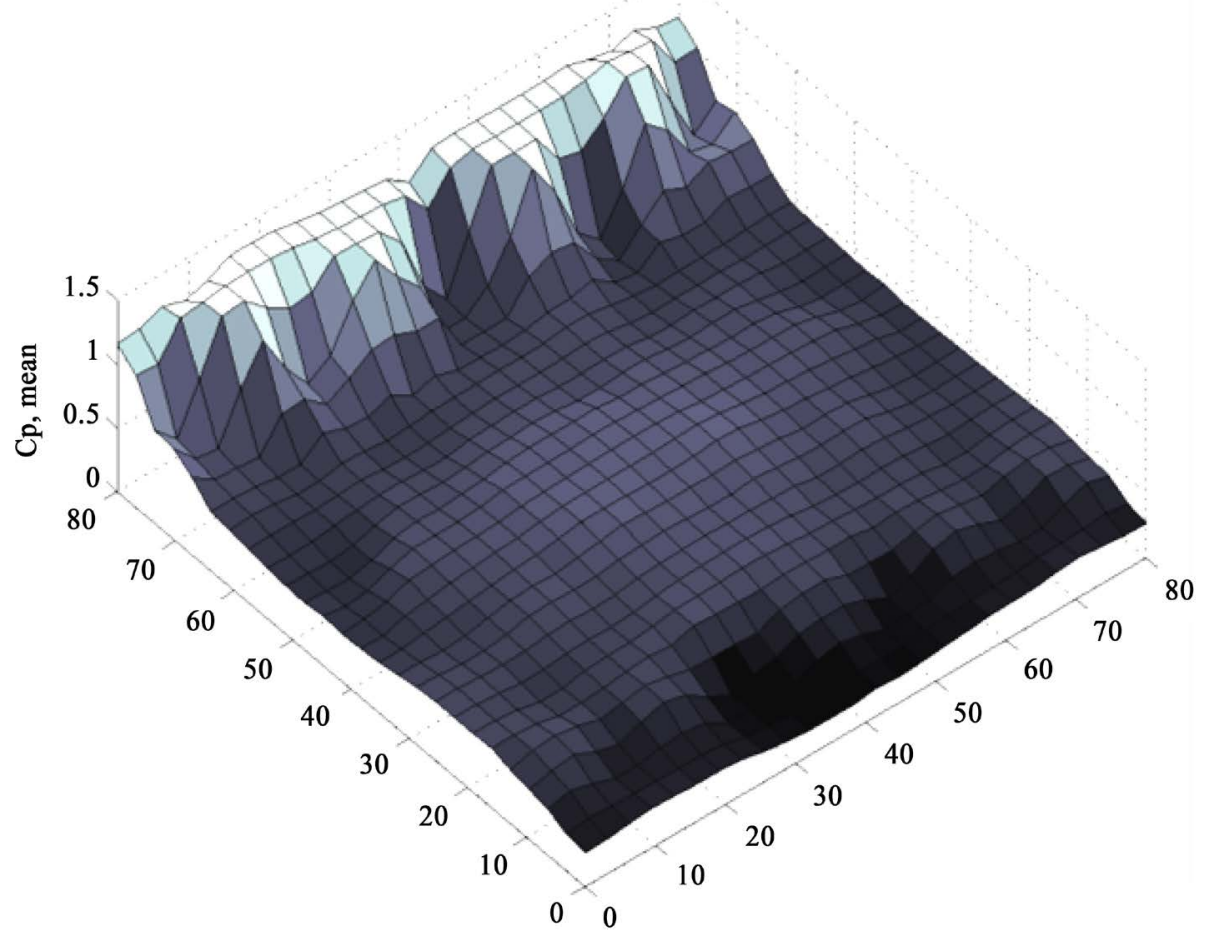

(c)

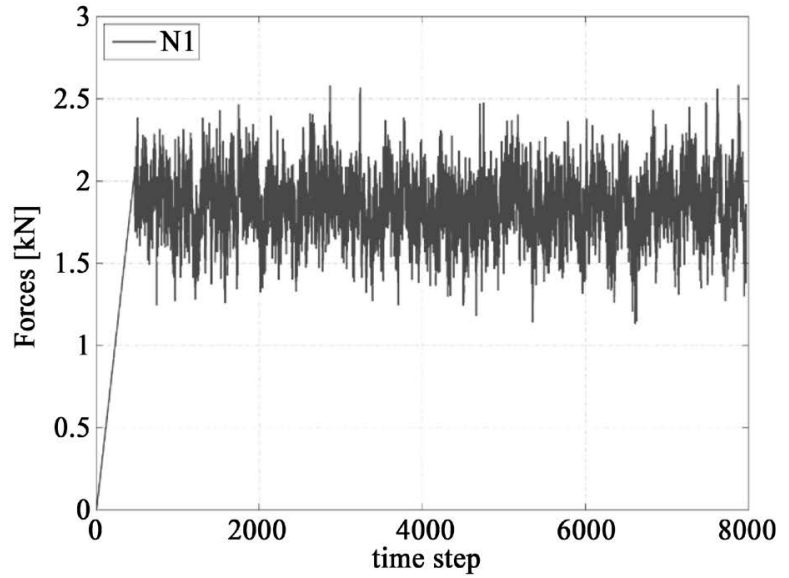

(d)

Figure 13. (a) Model in the wind tunnel; (b) Pressure coefficients map (mean values); (c) Pressure coefficients 3D mesh; (d) Typical forces time history.

dynamics; it is evaluated by the ratio between the difference of the local pressure and the undisturbed flow pressure, and undisturbed dynamic flow pressures are evaluated for each wind direction. Maximum $\left(\mathrm{C}_{\mathrm{p}, \max }\right)$, minimum $\left(\mathrm{C}_{\mathrm{p}, \min }\right)$ and mean values $\left(\mathrm{C}_{\mathrm{p}, \mathrm{m}}\right)$ of the pressure coefficients are extracted from the obtained pressure time histories. Subsequently, pressure coefficients maps were plotted. An example of Mean value of pressure coefficients map is shown in Figure 13(b). Minimum and maximum pressure coefficients have been calculated using a probabilistic method according to the Gumbel method (Gumbel, E.J., 1958) following the procedure proposed in (Cook N.J., Mayne J.R., 1979), (Cook N.J., Mayne J.R., 1980), associated with a probability of 22\% that it will be exceeded, as is done by Eurocode 1. For each pressure taps and for each model, the numerical procedure computes a pressure coefficients time history of 7504 points, a pressure coefficients matrix consisting of 7504 rows and a number of columns equal to the number of pressure taps. Moreover, for each model the procedure calculates three vectors containing the mean, maximum and minimum value of the pressure coefficient. 
In the following the numerical procedure described in this section will be named NPWDP (Numerical Procedure for the Wind tunnel Data Process).

\subsubsection{Data Exchange between Wind Tunnel and FEM Analysis}

In order to use experimental data to perfume FEM analyses, the same surface discretization between wind tunnel test model and FE model is necessary. Often that is impossible because the number of pressure taps used for each models is generally less than the FE model nodes. Also in this case it was happened. The mean value of pressure taps number used on the roof is 90, the number of nodes used in FEM analysis to describe the cable net is about 1700. A numerical procedure to extend the experimental data on the FEM mesh is necessary. There are to more used possibilities: the first is obtained overlapping the FEM mesh and the Thiessen polygons distribution, the same pressure coefficient is used for all FEM nodes that are surrounding by polygon. A second way is to estimate a mathematical procedure to interpolate the experimental data respect the FEM mesh. Both procedures were implemented. The first solution follows the following sequence: the polygons edges coordinates (Cartesian reference system) are determined; a scan of the FEM nodes coordinates is been done in order to check the proximity from the polygon edge. The nodes that have coordinates between the polygon minimum and maximum coordinates are in the polygon. The second procedure is more difficult. The first phase is the same to the previous solution, but during the scan a value of pressure coefficients for each node is assumed; the values is evaluated using the inverse distance weight (IDW) interpolation method (Borrough P.A., 1986; Greville T.N.E., 1969; Hohn M.E. (editor), 1998). An example of 3-dimensional pressure coefficients map obtained using this last method is shown in Figure 13(c). Using the pressure coefficients time history assumed for each FEM nodes, a wind loads time history is evaluate. In this case in order to obtain the structural response of a cable net under wind action, a localization of the net is necessary. A value of wind kinetic pressure and geographical aspects are chosen. A preliminary ramp is added by the load history, it has a length equal to about $1 \%$ of the history length. In Figure 13(d) an example of the load history is shown for a cable net center point (N1) (Shen S., Yang Q. 1999). In the following the numerical procedure described in this section will be named NPED (Numerical Procedure to Exchange Data). In Figure 14 the procedure is summarized [16]-[18].

\section{Wind-Structure Interaction with Time History Analysis}

In order to estimate cables nets structural response, Nonlinear Dynamic Analyses have to be performed. The analyses are conducted on the tested sample using numerical procedures implemented by Full Professor PieroD'Asdia staff; the procedures are merged in a main program in the following named TENSO. It isn't a commercial software and it was born as a set of numerical procedures and subroutines merged step by step from 1980. The description of these procedures has never been published.

\section{The Non Linear Structural Analysis Program}

The structural analysis program (in the following TENSO) is designed for static and dynamic analysis with step-by-step integration of nonlinear geometric three-dimensional structures. It contains cable and beam finite elements and permits the study of wind-structure interaction with generation of wind histories and simulation of aeroelastic phenomena (Crisfield M.A., 1991). Nonlinear static analysis were carried out with the wind action evaluated for mean, minimum and maximum pressure coefficients while nonlinear dynamic analysis were performed by applying wind action as a forces time history computed with the previously described numerical procedure. With TENSO is possible to compute parabolic cables in two ways: in the first case, the cable can be divided in an appropriate number of elements that are rectilinear cable, in the second case elastic catenary configuration or parabolic cable can be used. The first case is applicable only with nodal loads. Possible applications of this methodology are suspension bridges with a distance between the hangers sufficiently small compared to the cable span length, and cables nets with a small spacing between cables compared to the maximum span length. For this kind of structures the global stiffness matrix is updated for each load step through the assembly of stiffness matrices of the elements varied according to the strain found in the previous step. In this way the software takes into account the geometric nonlinearity of the structure. As regards to the beam finite element, it is possible to choose among a beam with a uniform or a variable section. For each case it is possible to introduce prestressing actions or tractions as well as thermal loads. In particular, the beam finite element with variable section provides the calculation of ten coefficients in order to describe the area variation and the moment of 


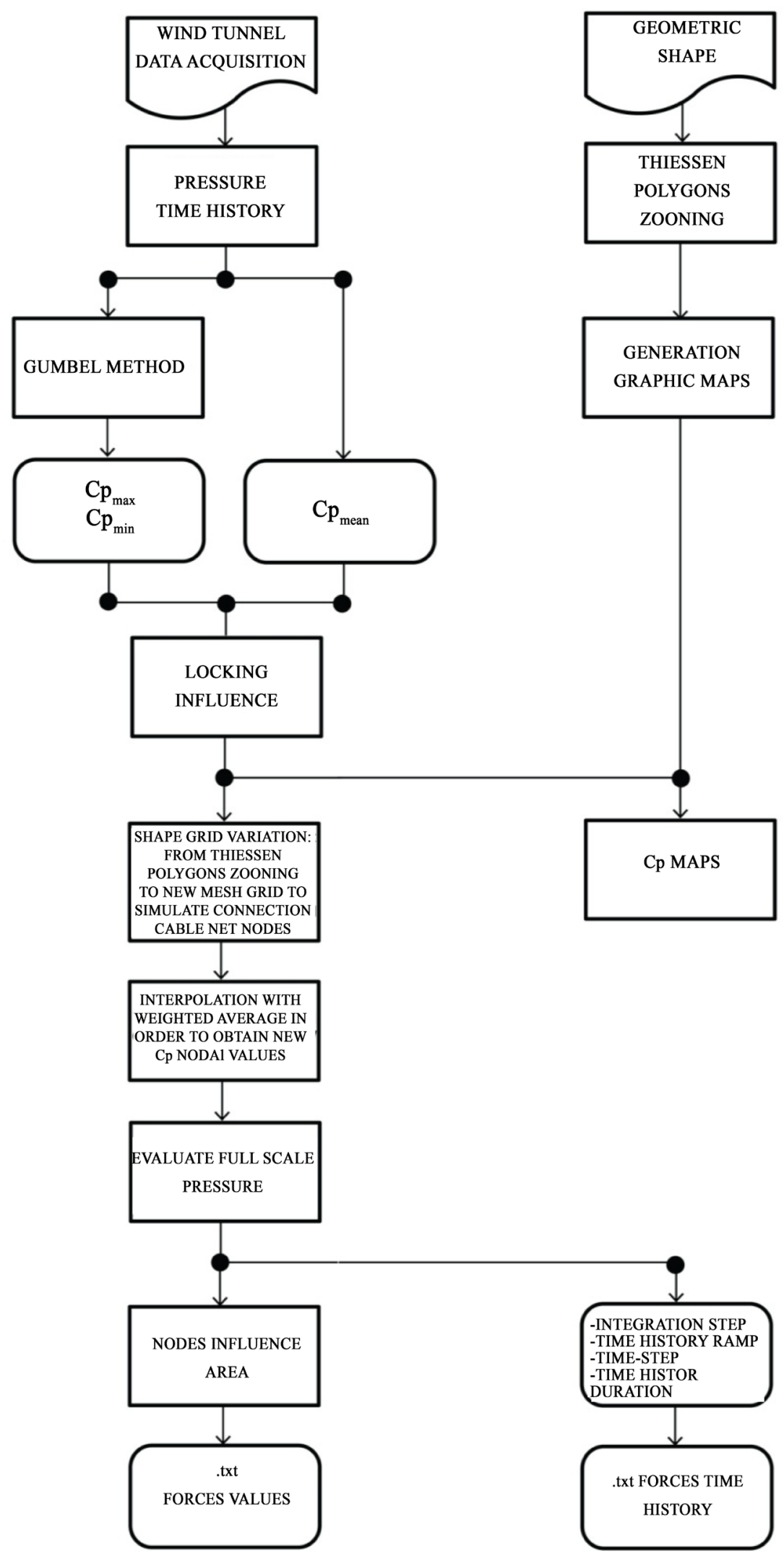

Figure 14. Flow chart of the wind tunnel data processing. 
inertia along the beam axis with a polynomial function. In order to evaluate the stiffness matrix, a numerical procedure based on the validity of the constitutive elastic low is used and six independent functions to describe the displacement field is used, too. At the beginning, the procedure computes the flexibility matrix of the element, applying a forces system and evaluating a displacement system using an algorithm that, step by step, computes the twelve conditions of kinematic compatibility and the balance evaluated in the previous step. The Gauss method is used for the numerical integration and Rayleigh damping is implemented. In the case investigated a value of $1 \%$ of structural damping is used; the value is in line with literature references. TENSO software simultaneously uses two solution methods to solve nonlinear analysis: the step by step incremental method and the interaction progressive method with the variable stiffness matrix as well as the secant method. The secant method can be thought of as a finite difference approximation of Newton's method.

In TENSO, secant method is used as a check method that permits to stop the analysis with a unbalanced solution. Using the step by step incremental method, nonlinear problem can be transformed in a succession of linear problems. Each calculation step stores loads and strains history evaluated during the previous step. For each analysis step, a small enough part of load $(\Delta P)$ necessary to ensure that is possible to use the elasticity method is applied. However, this simple and classical approach presents the difficulty to evaluate the exact dimension of load step and so the exact step of analysis. A non-appropriate chosen range can cause an inexact solution. In order to solve it, TENSO uses the method with the variable stiffness matrix; this method is a vector version of the Newton-Raphson modified method about nonlinear equation systems. The Newton-Raphson procedure guarantees convergence if and only if the solution at any iteration is close to the exact solution. Therefore, even without a path-dependent nonlinearity, the incremental approach (i.e., for subsequent load steps) is sometimes required in order to obtain a solution corresponding to the final load level. If the displacements are large, the product between the stiffness matrix, evaluated on the basis of the solution of the previous step and on the basis of the stresses, and the displacements vector, give the internal force vector, not equilibrate with the external forces vector according to Equation (20), where $K$ is the stiffness matrix, $\delta$ is the displacements vector, $\tilde{P}$ is the external forces vector; the difference between these two forces vectors represents the imbalance force vector, according to Equation (21), where $R$ is the residual vector and $P$ is the internal forces vector. In the following step, this vector is applied as an external load modifying the displacement vector with a residual value of displacements, according to Equation (22) where $\Delta \delta$ is residuals values of displacements to update the geometry, and so updating the structure geometry.

$$
\begin{gathered}
{[K]_{k+1}\{\delta\}_{k+1}=\{\tilde{P}\}_{k+1}} \\
{[R]_{k+1}=\left[\{P\}-\{\tilde{P}\}_{k+1}\right] \neq 0} \\
\{\Delta \delta\}_{k+1}=[K]_{k+1}^{-1}\{R\}_{k+1}
\end{gathered}
$$

In order to solve nonlinear dynamic analyses, TENSO uses the Newmark-beta method, a numerical integration method used to solve differential equations. It is used in finite element analysis to model dynamic systems. In order to illustrate the use of this family of numerical integration methods, the solution of a linear dynamic system have to be firstly considered. In 1962 Newmark's method in matrix notation was formulated, stiffness and mass proportional damping was added, and the need for iteration by introducing the direct solution of equations at each time step was eliminated. The time dimension is represented by a set of discrete points each a time increment apart. The system is solved at each of these points in time using as data the solution at a previous time. The procedure follows these subsequent phases: reading of initial boundary conditions; assembly of the stiffness matrix, setting $\beta$ and $\gamma$, Newmark method parameters that control the stability and the accuracy of the integration procedure. They are equal to $1 / 4$ and $1 / 2$ respectively; assembly of the vector forces; step by step calculation with iterative process and convergence check. For each integration step, a check of the solution precision is done in order to evaluate if it is necessary to modify the integration step dimension. Generally, a more precise solution needs a very small integration step and so a higher computational work. Unbalanced loads are evaluated according to the Newmark's algorithm, as the difference between the reactions and the applied external loads. In TENSO, a correction of this algorithm is implemented: the precision of solution is evaluated as a ratio between unbalanced loads and applied external loads for each unconstrained degree of freedom. At each integration step, unbalanced loads are added to the next load step, in order to obtain an optimal solution (Melchers R.E., 1987; Smith I. M., Griffiths D.V., 1982). In Figure 15, a global flow chart of TENSO is summarized. The main pro- 


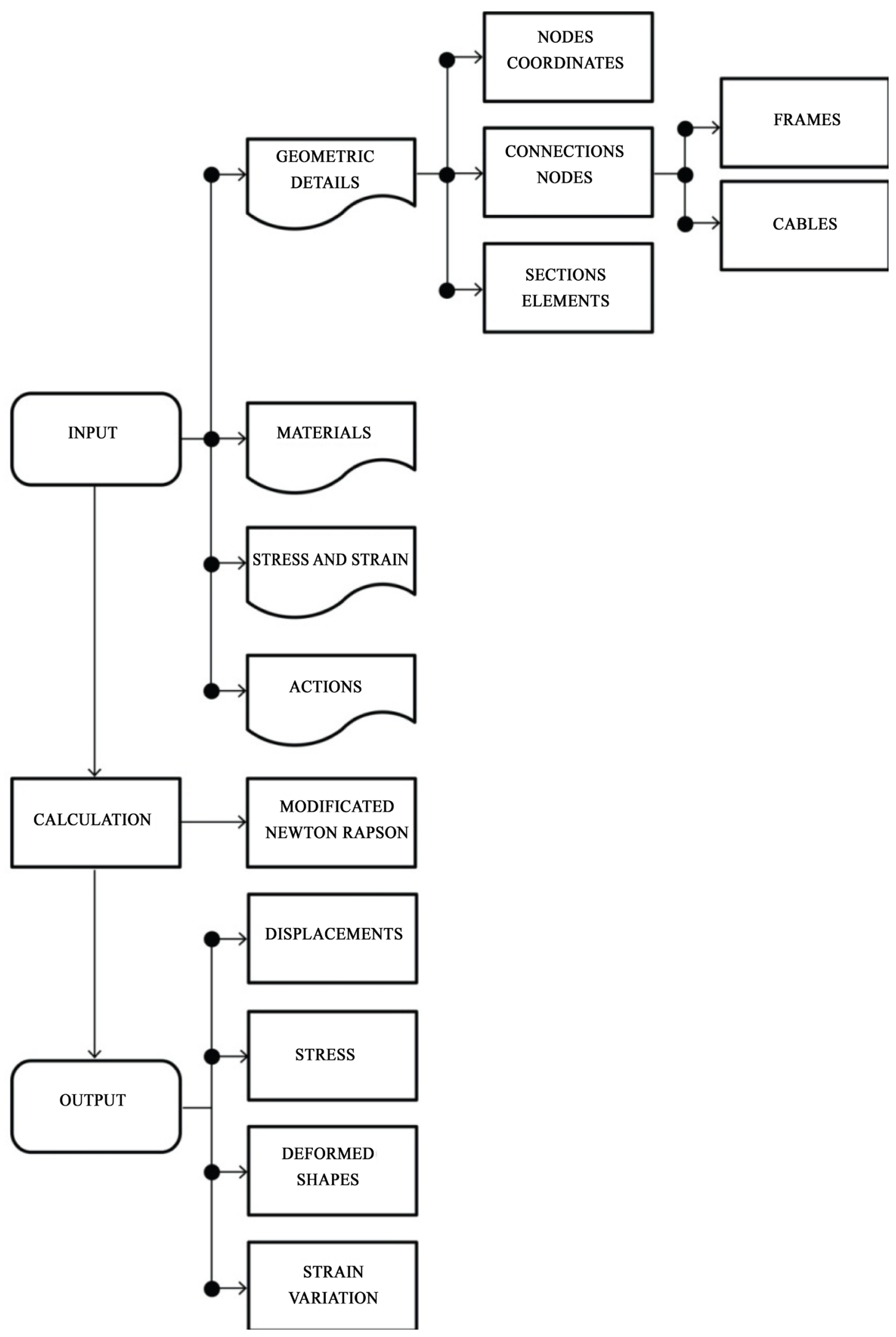

Figure 15. Nonlinear structural analysis program global flow chart. 
gram of the numerical procedures described in this section in the following will be named NPSA.

\section{Applications}

In order to demonstrate the validity of the procedure, two examples of different structures covered with cables net are described: the first one is a project proposal under review (2012) of a roller skates arena and the second is basketball arena; the last one is a project proposal for the renovation of an existent sports arena (2007).

\subsection{Project of a Roller Skates Arena}

The building designed is located in Pescara, middle Italy, and its purpose is to cover an existent open space used for roller skates competitions (Rizzo et al., 2014). The building has a circular plan and is covered with an Hyperbolic Paraboloid cables net roof. The most important geometrical parameters are summarized in Table 2. The horizontal traction (referring to Equation (11)) is absorbed by a series of external pillars and beams located around the building like are illustrated in Figure 17(c). In Figure 17(a) a global view of the urban contest and in Figure 17(b) a significant plan of the building are shown. The geometry chosen is one of the optimal geometries studied and described in Figure 16 and obtained by the procedure described in Section 2. In this phase of the project wind tunnel experiments results obtained with the parametric study described in Section 3 are sued. Of course after this first preliminary phase some specific wind tunnel experiments are necessary to study aerodynamic and aeroelastic effects. The pressure coefficients maps (Rizzo et al., 2011 and Rizzo, 2014) for two significant angles used to evaluate the wind action are shown in Figure 18. The wind direction of $0^{\circ}$ are parallel to the stabilizing cables and at contrary, the wind direction of $90^{\circ}$ are parallel to the load bearing cables.

A FEM model is analyzed and geometrical non-linear analyses are carried out in order to design the structural components of the building. The procedure described in Section 3 is followed to define the FEM models and some pictures are illustrated in Figure 19. The mechanical parameters are reported in Table 3, where $D$ is the externa diameter of the building, $A_{1}$ and $A_{2}$ are respectively the load bearing cables and the stabilizing cables, $\varepsilon_{1}$

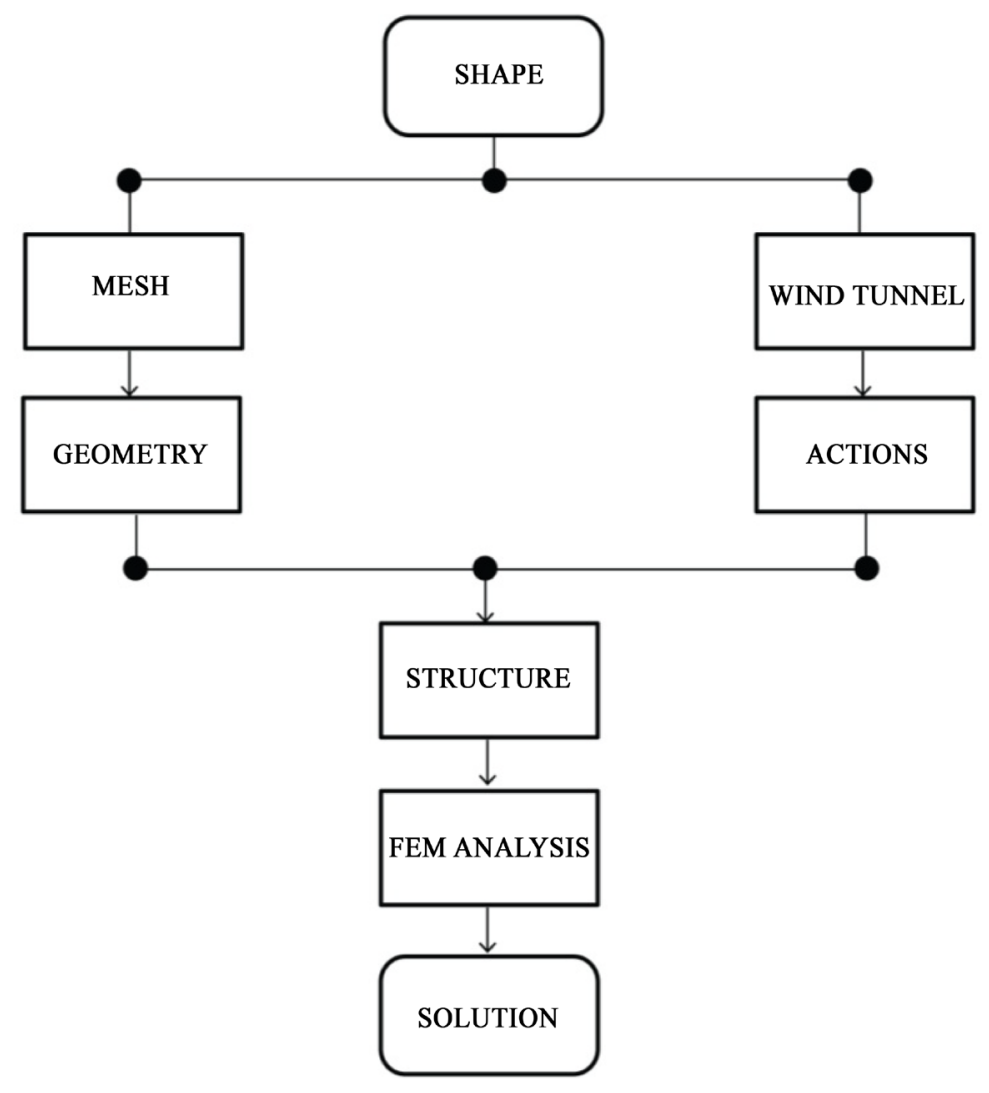

Figure 16. Procedure for the evaluation of tensile structure behaviour. 


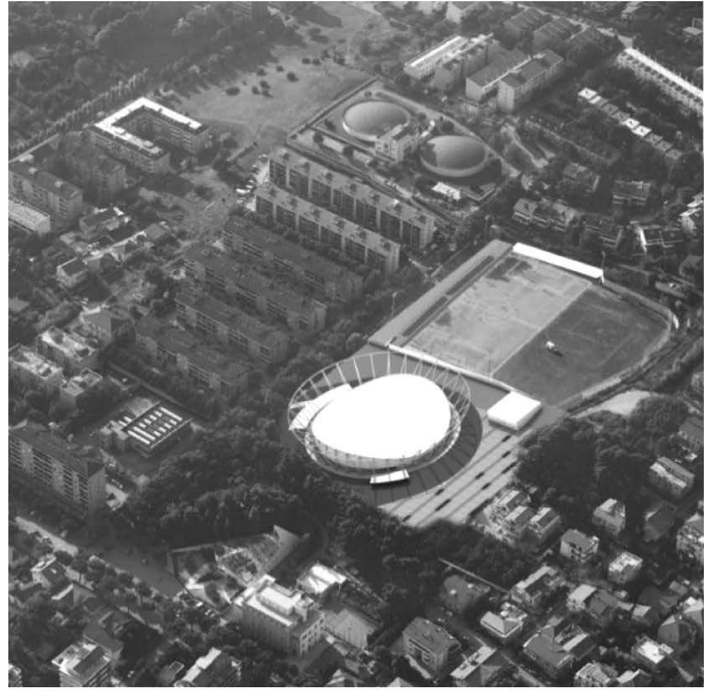

(a)

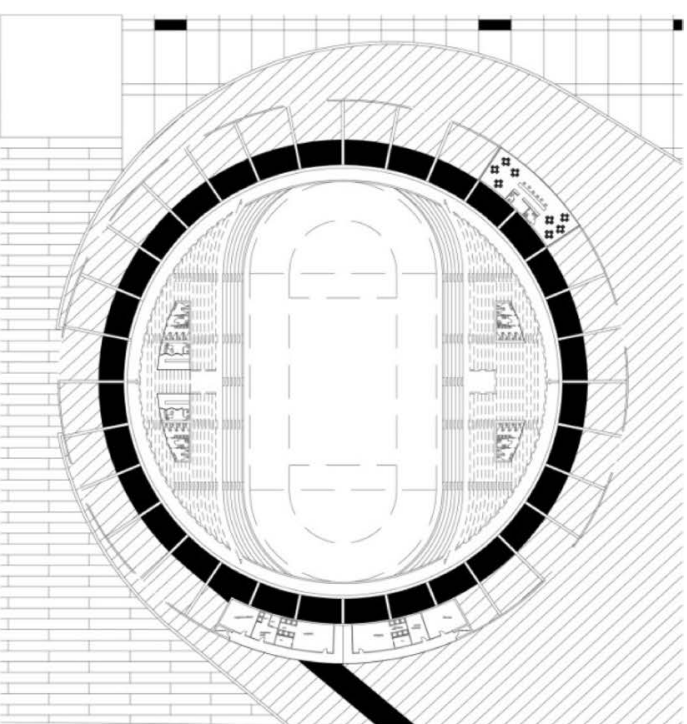

(b)

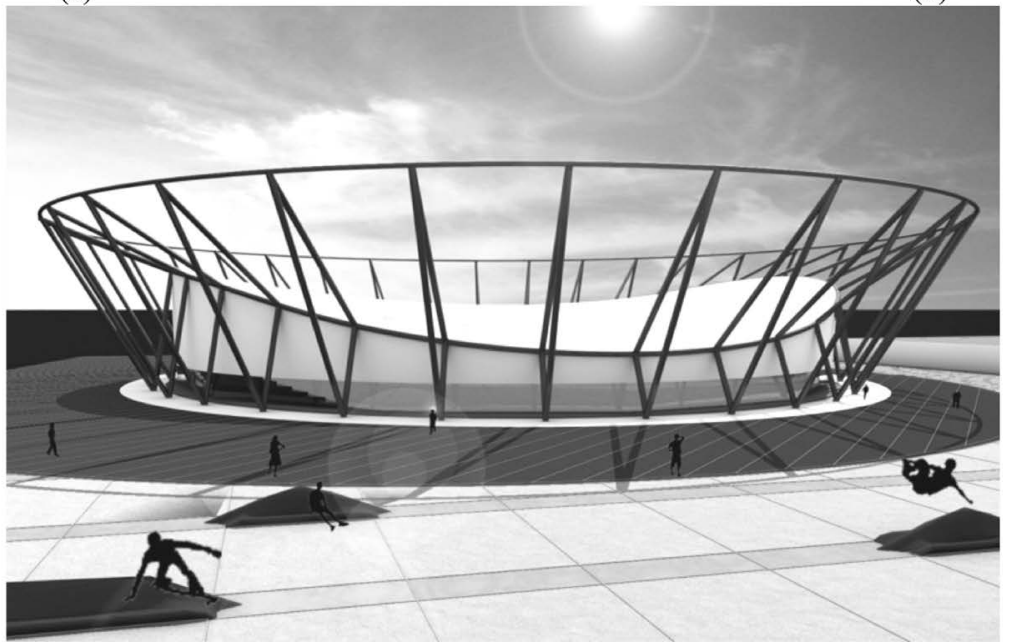

(c)

Figure 17. External views of the roller skates arena

and $\varepsilon_{2}$ are the strains cables for the load configuration number 1 (according to Section 2); $H_{b}$ is the drum high of the building, $H$ is equal to the $f_{1}+f_{2}$.

Using the numerical procedure described in Section 4, nonlinear analyses are carried out in order to study at first the structure deformation and its natural frequencies. Modal analyses and then a dynamic time history analysis are permed using the wind tunnel experiments results. Some results are illustrated in Figure 20 and Figure 21. In particular the first 9 modes shape are reported in Figure 20 and are listed in Table 4, the deformation under 0 wind action is reported in Figure 21. Observing the cables area values reported in Table 3 and considering that 40 load bearing cables and 40 stabilizing cables are used, we note that the structural weight of the roof is really low, the cables net weight is only $1.5 \times 10^{-2} \mathrm{kN}$ to square meters. One aspect ore is important to note: the first 9 modes are totally roof's mode and the periods are very high. This structures are particularly optimal in seismic zones because are flexible and ductile.

\subsection{Project of a Basketball Arena}

The project purpose is to substitute a truss structure used to cover a sport arena with a cable net structure. The advantages are many and the most important is the easy of maintenance. The great numbers of bolted nodes often require maintenance and the trusses require a very repetitive protective painting. The occasion to begin the 


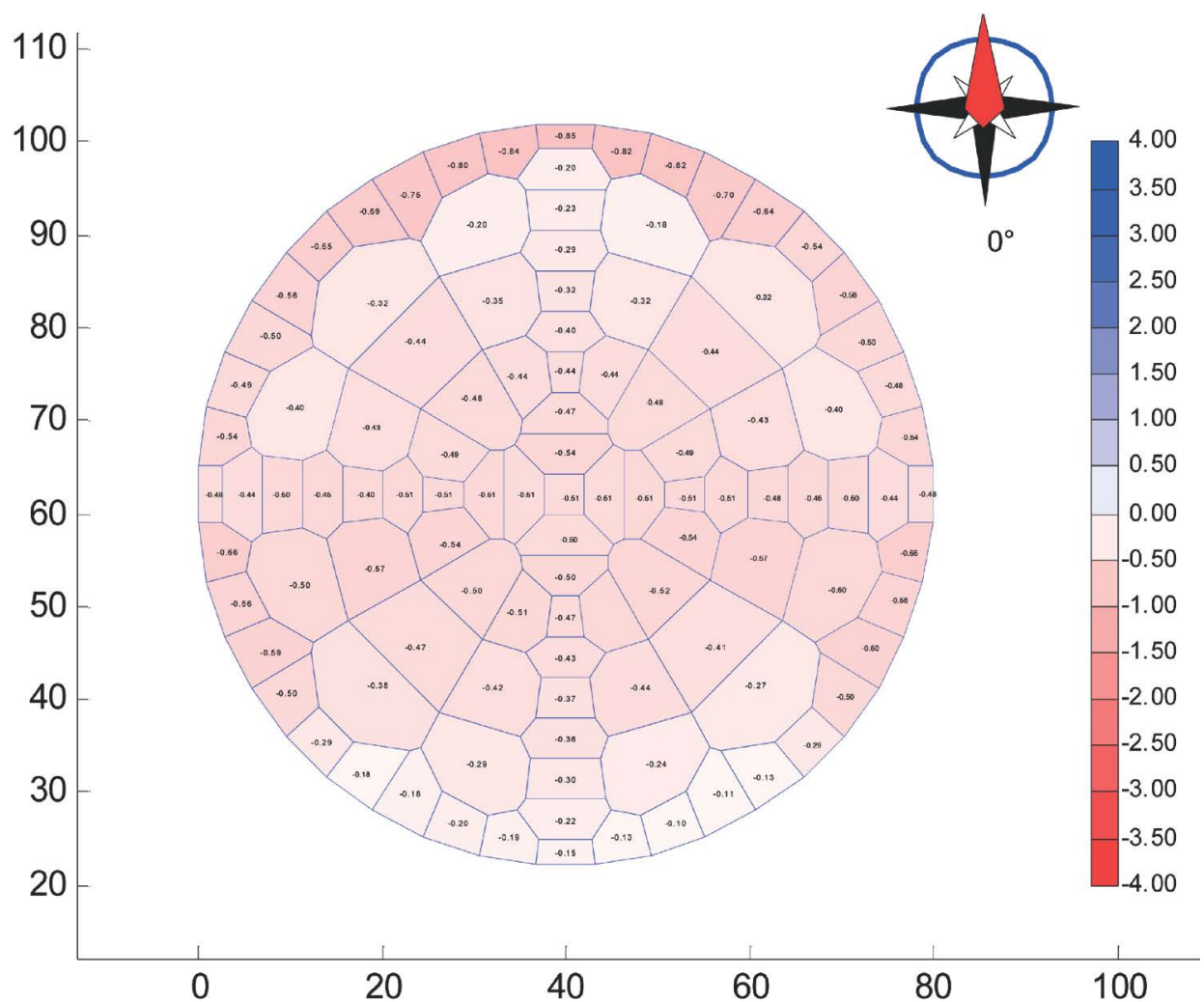

(a)

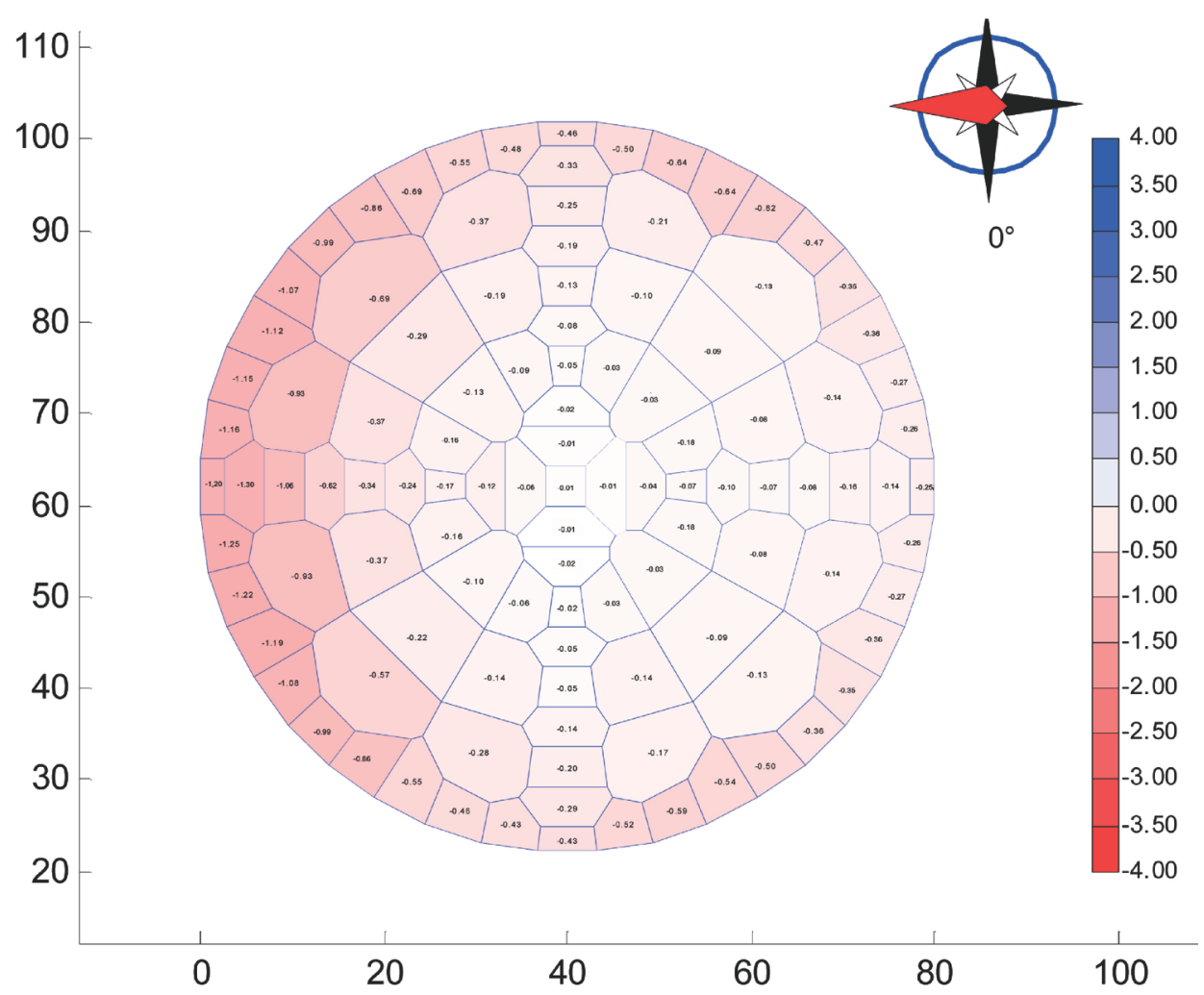

(b)

Figure 18. Pressure coefficients maps, (a) $0^{\circ}$ and (b) $90^{\circ}$. 


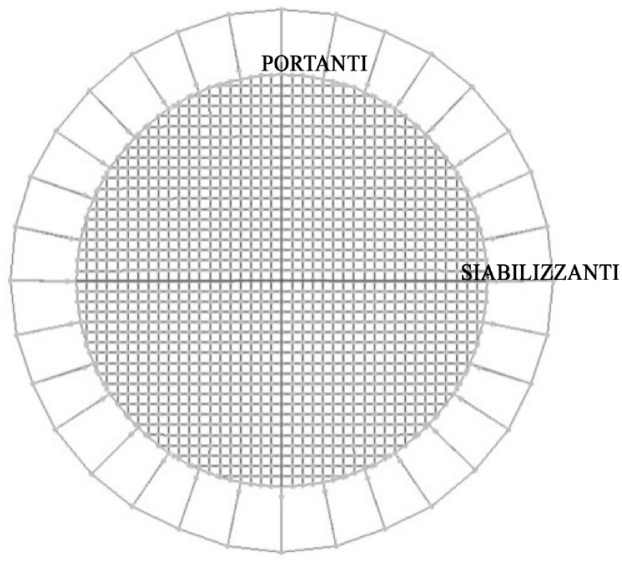

(a)

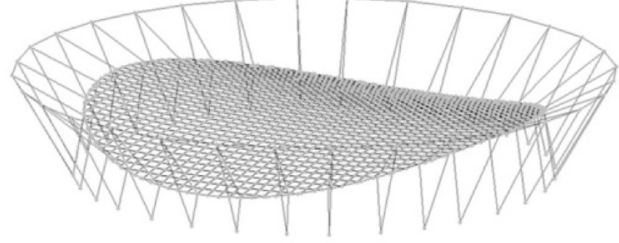

(b)

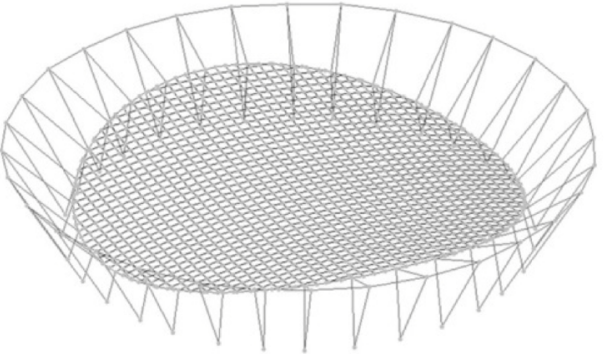

(c)

Figure 19. FEM models views.

Table 3. Geometrical and mechanical parameters.

\begin{tabular}{ccccccccc}
\hline$f_{1}(\mathrm{~m})$ & $f_{2}(\mathrm{~m})$ & $D(\mathrm{~m})$ & $H(\mathrm{~m})$ & $H_{b}(\mathrm{~m})$ & $A_{1}\left(\mathrm{~cm}^{2}\right)$ & $A_{2}\left(\mathrm{~cm}^{2}\right)$ & $\varepsilon_{1}(\%)$ & $\varepsilon_{2}(\%)$ \\
\hline 2.67 & 5.33 & 80.00 & 8.00 & 13.33 & 7.89 & 3.93 & 11.45 & 5.13 \\
\hline
\end{tabular}

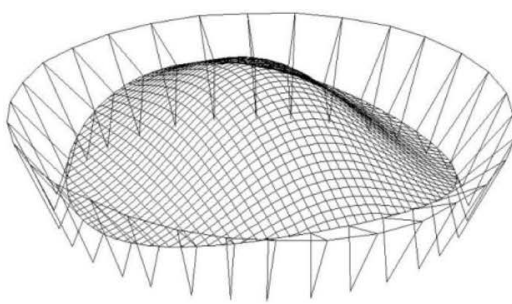

Mode $1^{\text {th }}$

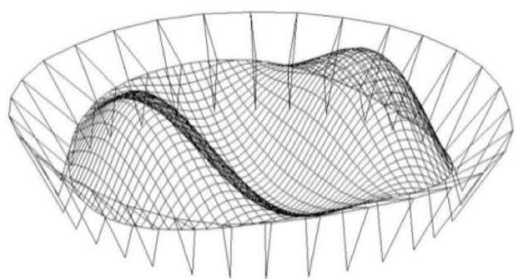

Mode $4^{\text {th }}$

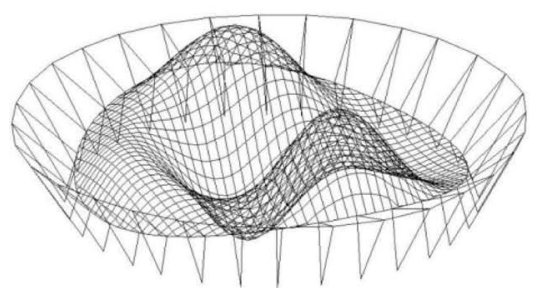

Mode $7^{\text {th }}$

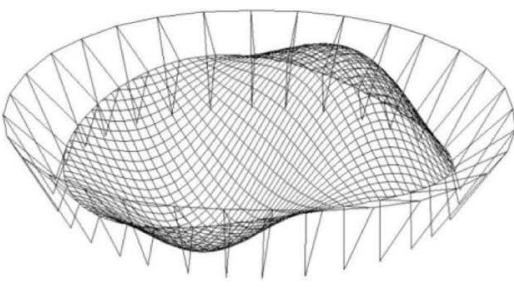

Mode $2^{\text {nd }}$

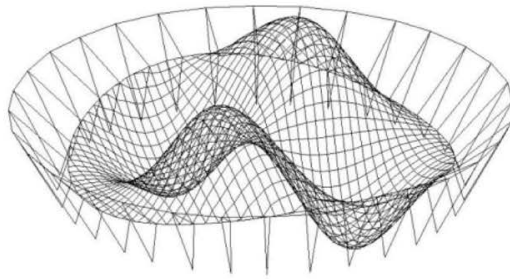

Mode $5^{\text {th }}$

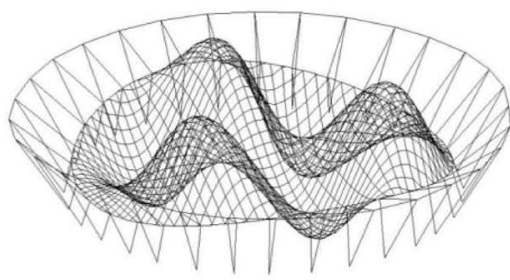

Mode $8^{\text {th }}$

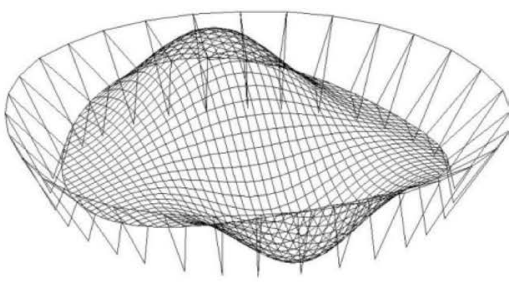

Mode $3^{\text {rd }}$

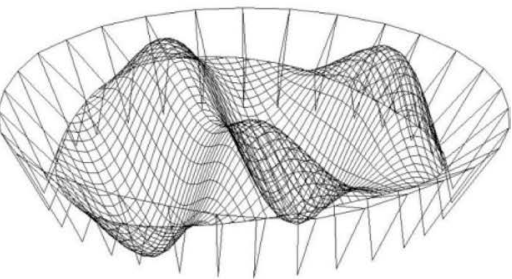

Mode $6^{\text {th }}$

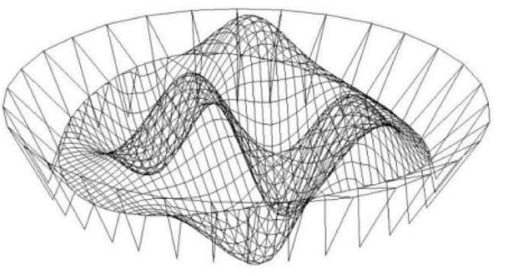

Mode $9^{\text {th }}$

Figure 20. Modes shape deformation. 
Table 4. Natural frequencies and periods.

\begin{tabular}{ccc}
\hline Mode & Frequency $(\mathrm{Hz})$ & Period (s) \\
\hline 1 & 0.149 & 6.679 \\
2 & 0.179 & 5.584 \\
3 & 0.215 & 4.647 \\
4 & 0.244 & 4.099 \\
5 & 0.254 & 3.933 \\
6 & 0.293 & 3.417 \\
7 & 0.296 & 3.377 \\
8 & 0.313 & 3.190 \\
9 & 0.340 & 2.939 \\
10 & 0.352 & 2.844 \\
\hline
\end{tabular}

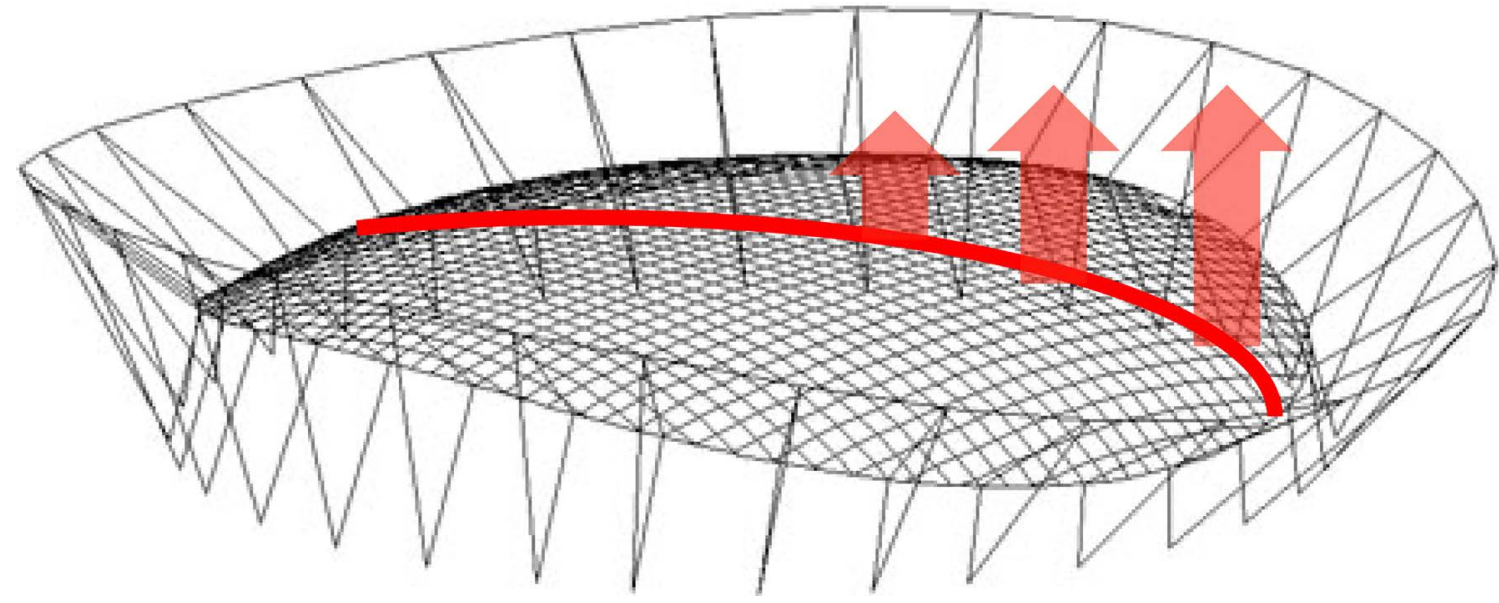

Figure 21. Upward deformation under $0^{\circ}$ wind action.

building renovation was the championship European basketball competition (2007) (Rizzo et al., 2012). The proposal is being evaluated by the municipality government for the future renovation.

The idea is to realize an external structure with a square plan in order to have a total open space into the arena. Some pictures of the actual structure and the future modification are illustrated in Figure 22. A square plane is chosen in order to respect the actual shape of the building and its urban contest. A series of stays are used to absorb the horizontal traction of the cables net, like is shown in Figure 22. In Table 5 the main geometrical parameters are listed and the cables areas and strains for the load configuration number 1 (according to Section 2).

The structural response is evaluated with Non-Linear analyses carried out with a FEM model modelled with the numerical procedure described in Section 3. Some pictures of the FEM model are shown in Figure 23. Using the numerical procedure described in Section 5 the Natural frequencies and displacements under loads combinations are evaluated, too. In Figure 24 the first 10 modes shape deformations are reported and in Table 6 their values are listed. It's interesting to note that the period is higher than the other geometry described in Section 7.1; it's caused by many reason, at first the plan geometry because the circular shape gives a more rigid border structure, at second the cables areas and strains are lower for this geometry, finally, this structure is also higher than the previous.

The wind action is applied on the FEM model like a series of time histories evaluated by wind tunnel experiments and a dynamic analysis is performed. In Figure 25 a view of the structure deformation under $90^{\circ}$ wind action is shown; it is interesting to note that this direction is particularly critic for Hyperbolic paraboloid cables net because the suction is higher than the other angles (Rizzo et al., 2011), so it is particularly important that the suction not decreases too the load bearing cables strain. In this case, like is reported in Table 7, the ratio between load bearing cables strains with and without wind is equal to 0.85 (reduction equal to 15\%). In Table 6 
the structural response in term of cables strains, traction and deformation variation, is reported; $T_{1}$ and $T_{2}$ are the cables traction evaluated according to the Equation (10), $\Delta f$ is the sag variation.

\section{Conclusion}

Every time that an experiment is processed, a great number of numerical procedures are programmed by researcher to control the process. These numerical procedures are often isolated and programmed again for every different case of study. An interesting goal is to create a free open domain where the numerical procedures evaluated are merged, added, modified by researchers with the aim to obtain a common space of use. With this purpose, the present paper described a methodology followed to prepare a wind tunnel test and to process results. Five different steps NPPD $\rightarrow$ NPGFM $\rightarrow$ NPWDP $\rightarrow$ NPED $\rightarrow$ NPSA give a one complete numerical procedure that can be expanded, modified or completed by everyone. In this specific case the subroutines can be modified to capture more and more different geometries or structural typologies with the aim to obtain a globalized virtual space of calculus. This paper is focused to wind tunnel tests because they are generally complex,

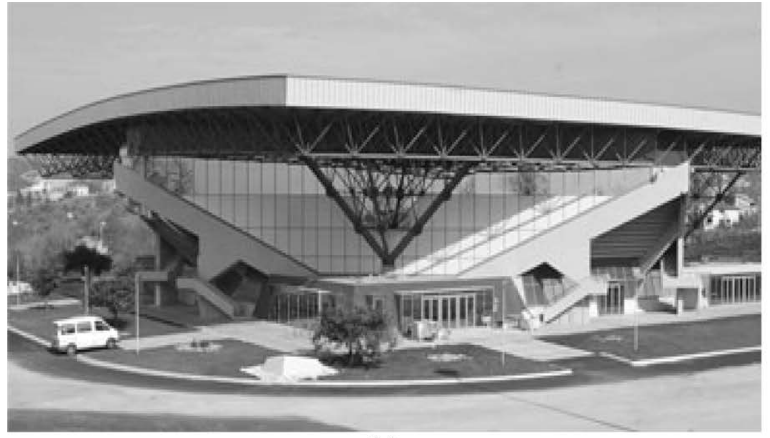

(a)

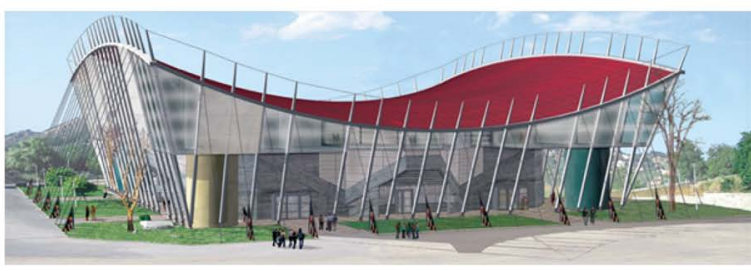

(b)

Figure 22. (a) External view of the actual structure; (b) Future modification of the building.

Table 5. Geometrical and mechanical parameters.

\begin{tabular}{cccccccccc}
\hline$f_{1}(\mathrm{~m})$ & $f_{2}(\mathrm{~m})$ & $L_{1}(\mathrm{~m})$ & $L_{2}(\mathrm{~m})$ & $H(\mathrm{~m})$ & $H_{b}(\mathrm{~m})$ & $A_{1}\left(\mathrm{~cm}^{2}\right)$ & $A_{2}\left(\mathrm{~cm}^{2}\right)$ & $\varepsilon_{1}(\%)$ & $\varepsilon_{2}(\%)$ \\
\hline 4.44 & 8.89 & 80.00 & 80.00 & 13.33 & 13.33 & 5.71 & 2.31 & 6.70 & 7.60
\end{tabular}

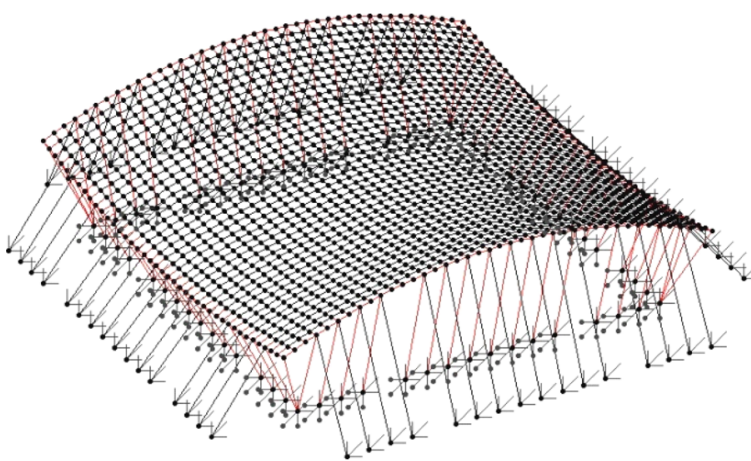

(a)

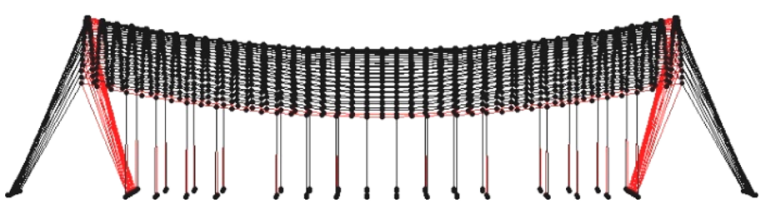

(b)

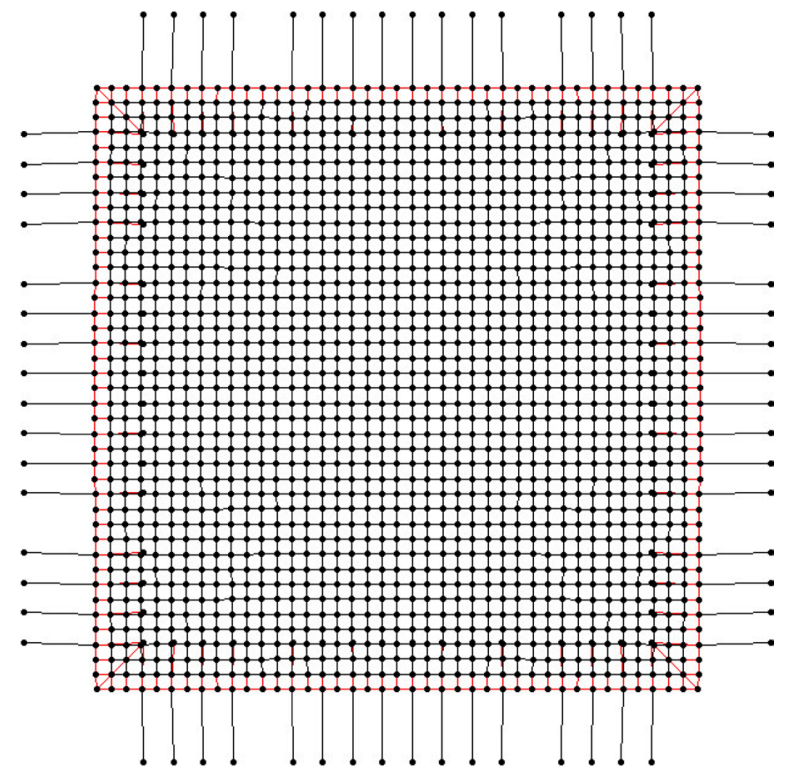

(c)

Figure 23. FEM model views. 


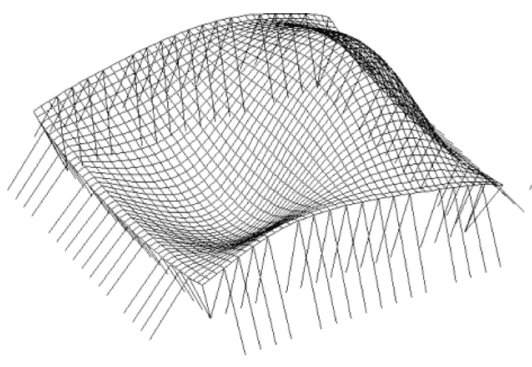

Mode $1^{\text {th }}$

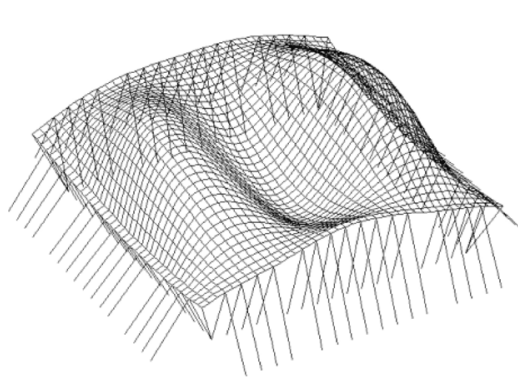

Mode $4^{\text {th }}$

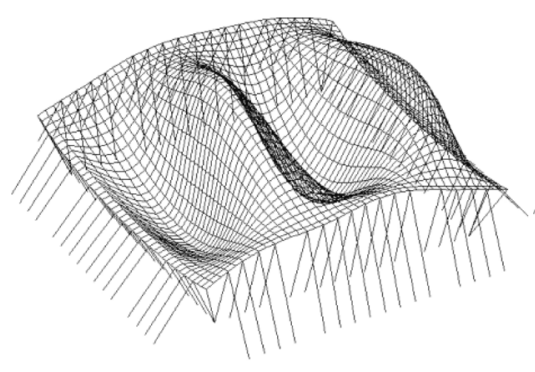

Mode $7^{\text {th }}$

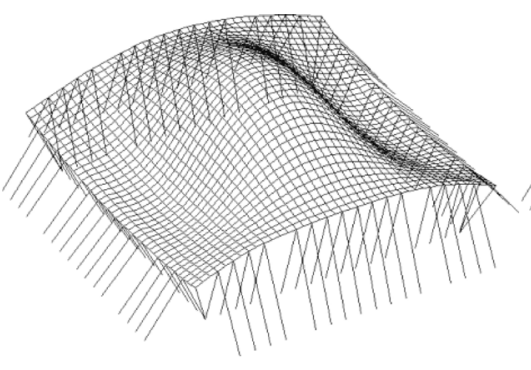

Mode $2^{\text {nd }}$

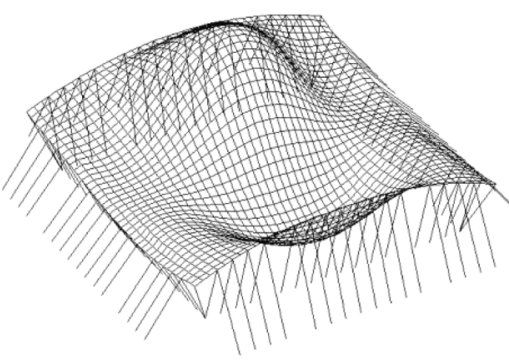

Mode $5^{\text {th }}$

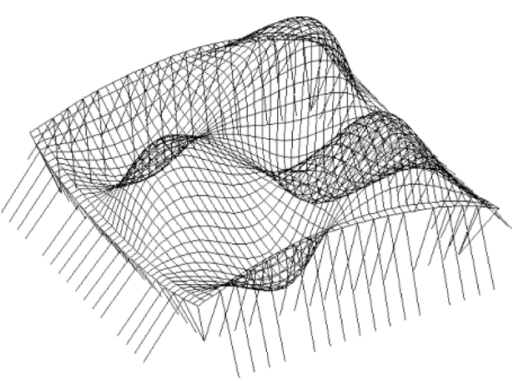

Mode $8^{\text {th }}$

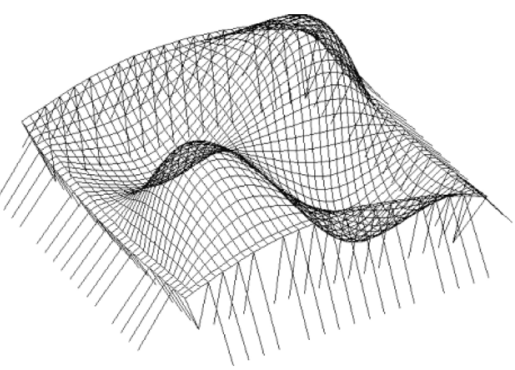

Mode $3^{\text {rd }}$

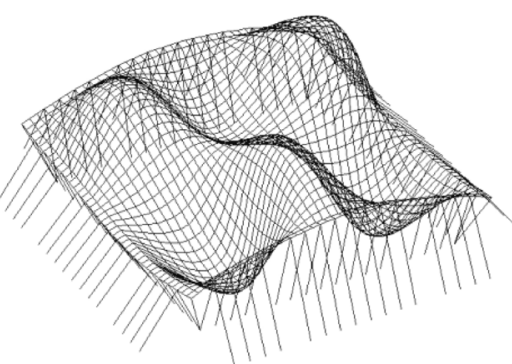

Mode $6^{\text {th }}$

Figure 24. Modes shape deformation.

Table 6. Natural frequencies and periods.

\begin{tabular}{ccc}
\hline Mode & Frequency $(\mathrm{Hz})$ & Period (s) \\
\hline 1 & 0.132 & 7.563 \\
2 & 0.154 & 6.509 \\
3 & 0.165 & 6.068 \\
4 & 0.180 & 5.566 \\
5 & 0.180 & 5.552 \\
6 & 0.202 & 4.944 \\
7 & 0.208 & 4.807 \\
8 & 0.233 & 4.299 \\
9 & 0.228 & 4.386 \\
10 & 0.247 & 4.047 \\
\hline
\end{tabular}

expansive and long test: a previous efficient and detailed preparation is necessary before and a great capacity to synthesize the results obtained is necessary after. With the methodology used the goal is obtained: in fact, at first the preliminary design procedure permits to choose the sample to test; at second the FEM generation procedure permits to obtain FE model for FEM analyses and a guide to construct wind tunnel test models; at third the 


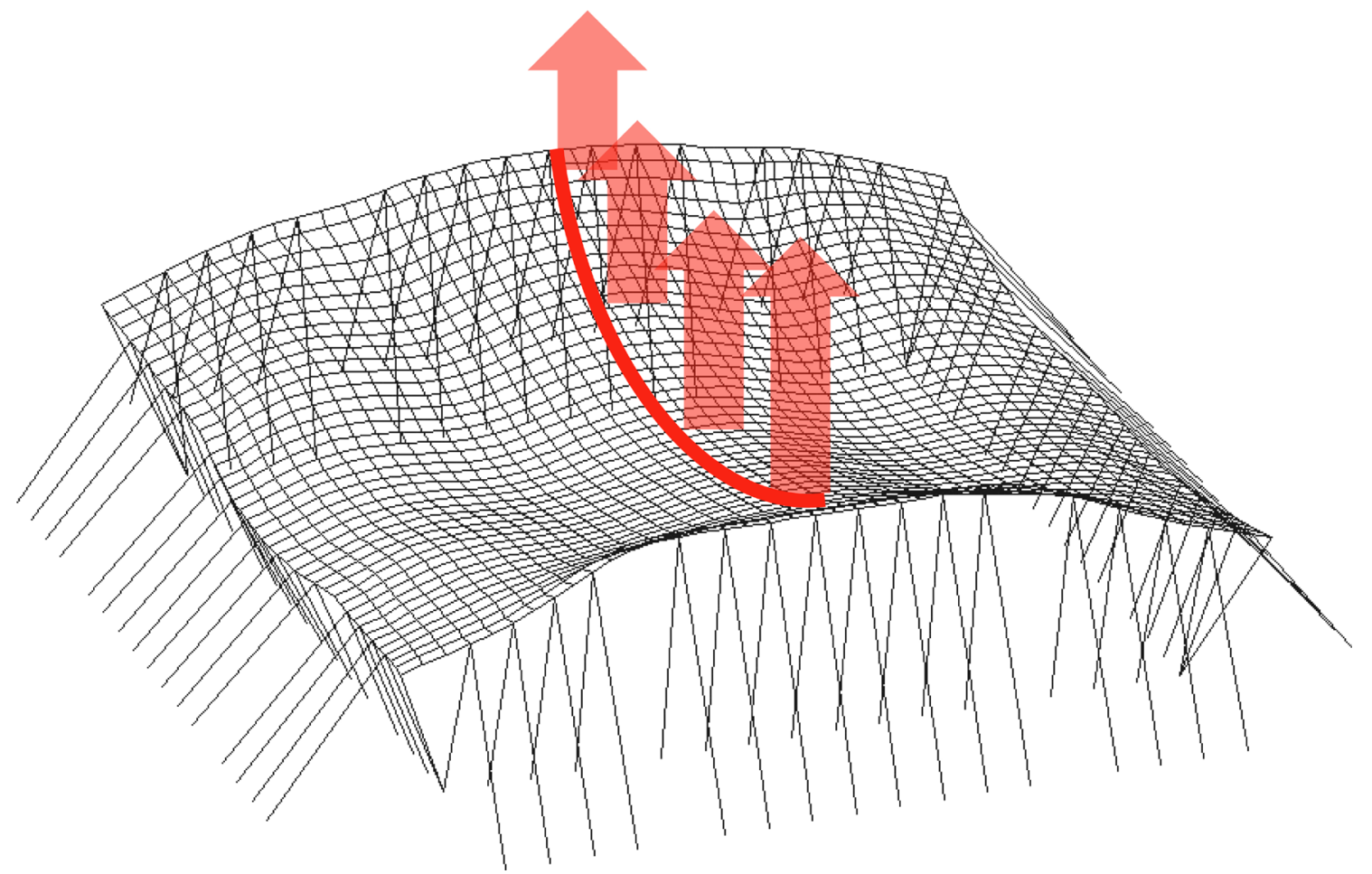

Figure 25. Upward deformation under $90^{\circ}$ wind action.

Table 7. Structural response.

\begin{tabular}{cccccccccccc}
\hline & \multicolumn{1}{c}{$\square$ (\%) } & \multicolumn{2}{c}{$\square$ (\%) } & \multicolumn{2}{c}{$T_{1}(\mathrm{KN})$} & \multicolumn{2}{c}{$T_{2}(\mathrm{KN})$} & \multicolumn{2}{c}{$\Delta f(\mathrm{~m})$} \\
\hline & $\max$ & $\min$ & $\max$ & $\min$ & $\max$ & $\min$ & $\max$ & $\min$ & $\max$ & $\min$ \\
\hline Comb.1 (Permanent loads) & 6.75 & 5.43 & 9.63 & 6.29 & 635.95 & 511.59 & 367.05 & 239.74 & -0.10 & $-3.4 \times 10^{-5}$ \\
Comb.2 Snow & 7.92 & 5.97 & 9.16 & 5.87 & 746.18 & 562.46 & 387.25 & 71.28 & -0.92 & $-1.0 \times 10^{-3}$ \\
Comb.3 Wind 0 & 6.00 & 4.85 & 9.86 & 6.98 & 659.51 & 456.94 & 375.81 & 266.04 & 0.36 & $1.7 \times 10^{-4}$ \\
Comb.3 Wind 90 & 5.78 & 3.92 & 10.14 & 6.87 & 657.62 & 463.83 & 386.87 & 262.04 & 0.30 & $1.5 \times 10^{-4}$ \\
\hline
\end{tabular}

processing data procedure permits to evaluate the experimental data and to prepare the input of the FEM analyses. Finally, the nonlinear structural analyses procedure permits to evaluate the structural response. The global flow chart is illustrated in Figure 16.

\section{Acknowledgements}

Special thanks to Full Professor PieroD’Asdia for the research coordination, to Engineer Massimiliano Lazzari for his for his collaboration in the planning process described in Section 2, to Engineer Fabrizio Fattor for his collaboration in the planning process described in Section 3, to Associate Professor Gianni Bartoli and the CRIACIV Wind tunnel boundary layer staff, in particular Engineer PhD Tommaso Massai and Lorenzo Procino, for the coordination of the wind tunnel tests and the numerical procedure programming described in Section 4, to Architect PhD Federica Speziale for the FEM analysis described in Section 5, finally to PieroD’Asdia, FabrizioFattor, Salvatore Noè and Luca Caracoglia for the calculation program described in Section 5.

\section{References}

[1] Rizzo, F. (2014) Aerodynamic of Tensile Structures. Silvana Editoriale, Milan.

[2] Rizzo, F., D’Asdia, P., Lazzari, M. and Procino, L. (2011) Wind Action Evaluation on Tension Roofs of Hyperbolic Paraboloid Shape. Engineering Structures, 33, 445-461. http://dx.doi.org/10.1016/j.engstruct.2010.11.001 
[3] Rizzo, F., D’Asdia, P., Ricciardelli, F. and Bartoli, G. (2012) Characterisation of Pressure Coefficients on Hyperbolic Paraboloid Roofs. Journal of Wind Engineering \& Industrial Aerodynamics, 102, 61-71. http://dx.doi.org/10.1016/j.jweia.2012.01.003

[4] Rizzo, F. (2012) Wind Tunnel Tests on Hyperbolic Paraboloid Roofs with Elliptical Plane Shapes. Engineering Structures, 45, 536-558. http://dx.doi.org/10.1016/j.engstruct.2012.06.049

[5] Rizzo, F., D’Asdia, P. and Speziale, F. (2012) FEM Analysis of Tension Structures with Experimental Wind Action. The 2012 International Conference on Advances in Wind and Structures (AWAS'12), Seoul, 26-30 August 2012, 33733381.

[6] Rizzo, F. and Sepe, V. (2015) Static Loads to Simulate Dynamic Effects of Wind on Hyperbolic Paraboloid Roofs with Square Plan. Journal of Wind Engineering \& Industrial Aerodynamics, 137, 46-57. http://dx.doi.org/10.1016/j.jweia.2014.11.012

[7] Rizzo, F., D’Asdia, P. and Speziale, F. (2014) Design of Hyperbolic Paraboloid Roofs with Circular and Elliptical Plan Shape. 13th National Conference of Wind Engineering, Geneva, 22-25 June 2014.

[8] Lewis, W.J. (2004) Tension Structures: Form and Behaviour. ASCE Standard.

[9] Majowiecki, M. (2004) Tensostrutture: Progetto e Verifica. Edizioni Crea, Milano. (In Italian)

[10] Melchers, R.E. (1987) Structural Reliability. Elley Horwood Ltd., UK.

[11] ASCE (American Society of Civil Engineers) (2005) Minimum Design Loads for Buildings and Other Structures. ASCE 7-05.

[12] Australian/New Zealand Standard (2002) Structural Design Actions; Part 2: Wind Actions. AS/NZS 1170.2:2002.

[13] Borrough, P.A. (1986) Principles of Geographical Information Systems for Land Resources Assessment. Oxford University Press, Oxford.

ESRI (1996) ArcView Spatial Analyst Manual (Ver. 3.0).

[14] CNR (National Research Council of Italy) (2011) CNR-DT 207/2008 — Guide for the Assessment of Wind Actions and Effects on Structures.

[15] CEN (Comité Européen de Normalisation) (2005) EN 1991-1-4: Eurocode 1: Actions on Structures—Part 1-4: General Actions-Wind Actions.

[16] Crisfield, M.A. (1991) Non-Linear Finite Element Analysis of Solids and Structures. Vol. 1, John Wiley \& Sons, Hoboken.

[17] Greville, T.N.E., Ed. (1969) Theory and Applications of Spline Functions. Proceedings of an Advanced Seminar, Madison, 7-9 October 1968, Academic Press, New York.

[18] Smith, I.M. and Griffiths, D.V. (1982) Programming the Finite Element Method. John Wiley \& Sons, Hoboken.

[19] Cook, N.J. and Mayne, J.R. (1979) A Novel Working Approach to the Assessment of Wind Loads for Equivalent Static Design. Journal of Wind Engineering \& Industrial Aerodynamics, 4, 149-164. http://dx.doi.org/10.1016/0167-6105(79)90043-6

[20] Cook, N.J. and Mayne, J.R. (1980) A Refined Working Approach to the Assessment of Wind Loads for Equivalent Static Design. Journal of Wind Engineering \& Industrial Aerodynamics, 6, 125-137. http://dx.doi.org/10.1016/0167-6105(80)90026-4

[21] Elashkar, I. and Novak, M. (1983) Wind Tunnel Studies of CABLE ROOFS. Journal of Wind Engineering \& Industrial Aerodynamics, 13, 407-419. http://dx.doi.org/10.1016/0167-6105(83)90160-5

[22] Gumbel, E.J. (1958) Statistic of Extremes. Columbia University Press, Columbia. Mayne, J.R. (1978) On Design Procedures for Wind Loading. Building Research Establishment, Garston.

[23] Gumbel, E.J. (1958) Statistic of Extremes. Columbia University Press, Columbia. Lieblein J. (1974) Efficient Methods of Extreme Value Methodology. Report 74-602, National Bureau of Standards, Washington DC.

[24] Shen, S. and Yang, Q. (1999) Wind-Induced Response Analysis and Wind-Resistant Design of Hyperbolic Paraboloid Cable Net Structures. International Journal of Space Structures, 14, 57-65. http://dx.doi.org/10.1260/0266351991494696

[25] Simiu, E. and Scanlan, R.H. (1986) Wind Effects on Structures. John Wiley \& Sons, Hoboken. 